ISSN 2072-6651

www.mdpi.com/journal/toxins

Article

\title{
Metabolic and Hematological Consequences of Dietary Deoxynivalenol Interacting with Systemic Escherichia coli Lipopolysaccharide
}

\author{
Erik Bannert ${ }^{1, \dagger}$, Tanja Tesch ${ }^{1, \dagger}$, Jeannette Kluess ${ }^{1, *}$, Jana Frahm ${ }^{1}$, Susanne Kersten ${ }^{1}$, \\ Stefan Kahlert ${ }^{2}$, Lydia Renner ${ }^{2}$, Hermann-Josef Rothkötter ${ }^{2}$ and Sven Dänicke ${ }^{1}$ \\ 1 Institute of Animal Nutrition, Friedrich-Loeffler Institute (FLI), \\ Federal Research Institute for Animal Health, Bundesallee 50, Braunschweig 38116, Germany; \\ E-Mails: Erik.Bannert@fli.bund.de (E.B.); Tanja.Tesch@fli.bund.de (T.T.); \\ Jana.Frahm@fli.bund.de (J.F.); Susanne.Kersten@fli.bund.de (S.K.); \\ Sven.Dänicke@fli.bund.de (S.D.) \\ 2 Institute of Anatomy, Otto-von-Guericke University Magdeburg, Leipziger Str. 44, \\ Magdeburg 39120, Germany; E-Mails: stefan.kahlert@med.ovgu.de (S.K.); \\ lydia.renner@med.ovgu.de (L.R.); hermann-josef.rothkoetter@med.ovgu.de (H.-J.R.) \\ $\dagger$ These authors contributed equally to this work. \\ * Author to whom correspondence should be addressed; E-Mail: jeannette.kluess@fli.bund.de; \\ Tel.: +49-531-5804-4101; Fax: +49-531-5804-4299.
}

Academic Editor: Jiujiang Yu

Received: 11 September 2015 / Accepted: 4 November 2015 / Published: 16 November 2015

\begin{abstract}
Previous studies have shown that chronic oral deoxynivalenol (DON) exposure modulated Escherichia coli lipopolysaccharide (LPS)-induced systemic inflammation, whereby the liver was suspected to play an important role. Thus, a total of 41 barrows was fed one of two maize-based diets, either a DON-diet (4.59 mg DON/kg feed, $n=19)$ or a control diet (CON, $n=22$ ). Pigs were equipped with indwelling catheters for pre- or post-hepatic (portal vs. jugular catheter) infusion of either control $(0.9 \% \mathrm{NaCl})$ or LPS $(7.5 \mu \mathrm{g} / \mathrm{kg} \mathrm{BW})$ for $1 \mathrm{~h}$ and frequent blood sampling. This design yielded six groups:

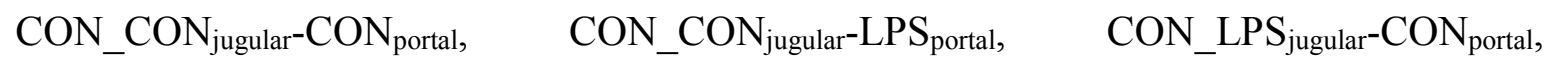
DON_CON jugular-CON $_{\text {portal }}$, DON_CON jugular $_{-} \mathrm{LPS}_{\text {portal }}$ and DON_LPS jugular-CON $_{\text {portal. }}$ Blood samples were analyzed for blood gases, electrolytes, glucose, $\mathrm{pH}$, lactate and red hemogram. The red hemogram and electrolytes were not affected by DON and LPS. DON-feeding solely
\end{abstract}


decreased portal glucose uptake $(p<0.05)$. LPS-decreased partial oxygen pressure $\left(p \mathrm{O}_{2}\right)$ overall $(p<0.05)$, but reduced $p \mathrm{CO}_{2}$ only in arterial blood, and DON had no effect on either. Irrespective of catheter localization, LPS decreased $\mathrm{pH}$ and base-excess $(p<0.01)$, but increased lactate and anion-gap $(p<0.01)$, indicating an emerging lactic acidosis. Lactic acidosis was more pronounced in the group DON_LPS jugular-CON portal than in CON-fed counterparts $(p<0.05)$. DON-feeding aggravated the porcine acid-base balance in response to a subsequent immunostimulus dependent on its exposure site (pre- or post-hepatic).

Keywords: swine; deoxynivalenol; E. coli lipopolysaccharides; endotoxin; sepsis; blood gas; metabolism; glucose; inflammatory response

\section{Introduction}

Due to its dependency on moderate climate conditions and its resistance to processing the Fusarium toxin, deoxynivalenol (DON) can be often found in toxicologically-relevant concentrations in cereals in temperate climate zones [1]. It is of special importance in pig production due to the high susceptibility of pigs, causing reduced feed intake and live weight gain, resulting in considerable economic losses [2-4].

Several studies indicate that DON influences the systemic inflammatory response. The toxin exerts immune modulatory effects on blood leukocytes depending on the dose and frequency of exposure. Different studies have shown that a low dose exposure to Fusarium toxins has an immune-stimulating effect due to an upregulation of transcriptional and post-transcriptional expression of cytokines, chemokines and inflammatory genes, whereas a high dose exposure has an immune-suppressive effect (reviewed in [5]). It has further been shown that exposure to DON causes an altered immune response [6-8] and liver cell metabolism $[9,10]$ to a subsequent lipopolysaccharide (LPS) challenge in vitro and in vivo.

Lipopolysaccharides form the major component of the outer cell membrane of Gram-negative bacteria and are responsible for the onset of an inflammatory response in the case of systemic LPS entry [11]. Triggering similar (immune biological) pathways, a variety of infectious pathogens, such as Gram-positive and Gram-negative bacteria, viruses and fungi, leads to identical clinical sequelae commonly described with the term sepsis $[12,13]$. Since infections with Gram-negative bacteria contribute to a substantial part of the sepsis cases worldwide, LPS-induced systemic inflammation is a well-established sepsis model in animals and humans [11,14-16].

On a systemic level, the recognition of LPS by the immune system causes the release of pro-inflammatory cytokines. This leads to inflammation, apoptosis, causing endothelial dysfunction, and microcirculation thrombosis, resulting in perfusion heterogeneity and microcirculatory failure [12]. Clinically, these alterations manifest themselves in a variety of symptoms, such as hypothermia or hyperthermia, tachycardia, tachypnea, edema, central nervous dysfunction, leukocytosis and leukopenia $[11,16,17]$. In concurrence with a pronounced inflammatory response, blood analysis reveals often lactic acidosis [18-20], along with either dysglycemia depending on the stage of disease [17,18,21]. 
As DON can be ubiquitous in cereals and pigs might be sub-acutely exposed on the one hand, while LPS is always present in the environment and commensal intestinal microbiota, on the other hand, pigs might be often co-exposed to both toxins at the same time. The liver possesses a central role in LPS detoxification [22,23] or in metabolic and immunological homeostasis [24] of, and it has been shown before that chronic exposure to DON leads to altered liver cell metabolism $[9,10]$. We hypothesized that liver metabolism and hematological variables are altered in chronically-DON-fed animals during a subsequent LPS stimulus. An increase in systemic LPS can either be a consequence of a systemic infection [17] or an increased passage from portal-drained viscera [25]. In order to simulate these pathways of systemic LPS entrance and the consequences of a possible hepatic first-pass effect, we infused LPS pre- or post-hepatically. Arterial, jugular and portal blood metabolic variables were assessed to evaluate the role of the liver in this pathogenesis as a consequence of LPS infusion.

\section{Results}

\subsection{Red Hemogram}

Generally, neither DON nor LPS treatment influenced red hemogram variables irrespective of infusion site, and all values were always in their respective physiological range [26] (Table 1). Slight fluctuations combined with small variances in all measures contributed to significant time, catheter and group $\times$ catheter $\times$ time effects (Table 1$)$.

Table 1. Effect of chronic enteral deoxynivalenol (DON) exposure and pre- or post-hepatic E. coli lipopolysaccharide (LPS) infusion on arterial, venous or portal red hemogram in pigs.

\begin{tabular}{ccccccc}
\hline \multirow{2}{*}{ Parameter } & \multirow{2}{*}{$\begin{array}{c}\text { Overall } \\
\text { Mean }\end{array}$} & \multirow{2}{*}{ PSEM } & \multicolumn{4}{c}{$\boldsymbol{p}$-Values } \\
\cline { 5 - 7 } & 5.57 & 0.24 & 0.306 & 0.299 & $<\mathbf{0 . 0 0 1}$ & 0.467 \\
$\mathrm{RBC}\left(\times 10^{6} \mathrm{cellls} / \mu \mathrm{L}\right)$ & 30.44 & 1.40 & 0.629 & $\mathbf{0 . 0 0 6}$ & $<\mathbf{0 . 0 0 1}$ & 0.415 \\
$\mathrm{Hct}(\%)$ & 10.82 & 0.49 & 0.618 & $0.073^{\mathrm{T}}$ & $<\mathbf{0 . 0 0 1}$ & 0.279 \\
$\mathrm{Hgb}(\mathrm{g} / \mathrm{dL})$ & 54.78 & 1.26 & 0.632 & $<\mathbf{0 . 0 0 1}$ & 0.981 & $\mathbf{0 . 0 3 6}$ \\
$\mathrm{MCV}(\mathrm{fL})$ & 19.48 & 0.52 & 0.602 & 0.144 & $<\mathbf{0 . 0 0 1}$ & $0.080^{\mathrm{T}}$ \\
$\mathrm{MCH}(\mathrm{g} / \mathrm{dL})$ & 35.56 & 0.52 & 0.554 & $\mathbf{0 . 0 0 1}$ & $<\mathbf{0 . 0 0 1}$ & 0.429 \\
$\mathrm{MCHC}(\mathrm{g} / \mathrm{dL})$ & &
\end{tabular}

Notes: $\mathrm{RBC}=$ red blood cells (reference: $5.5-8.5 \times 10^{6}$ cells $/ \mu \mathrm{L}^{1}$ ); Hct $=$ hematocrit (reference: $33 \%-45 \%{ }^{1}$ ); $\mathrm{Hgb}=$ hemoglobin (reference: $10.16-14.03 \mathrm{~g} / \mathrm{dL}^{1}$ ); $\mathrm{MCV}=$ mean corpuscular volume (reference: $50-65 \mathrm{fL}^{1}$ ); $\mathrm{MCH}=$ mean corpuscular hemoglobin (reference: $17-21 \mathrm{~g} / \mathrm{dL}^{1}$ ); $\mathrm{MCHC}=$ mean corpuscular hemoglobin concentration (reference: $\left.30-35 \mathrm{~g} / \mathrm{dL}^{1}\right) ;{ }^{1}=[26]$; references in venous blood; ${ }^{\mathrm{T}}=$ trend $(p \leq 0.10) ; \mathrm{PSEM}=$ pooled standard error of means. Barrows were either fed a DON contaminated ration (DON; $4.59 \mathrm{mg} / \mathrm{kg}$ feed) or control feed (CON) during 29 days. Infusion groups were divided as follows: pre-hepatic LPS infusion

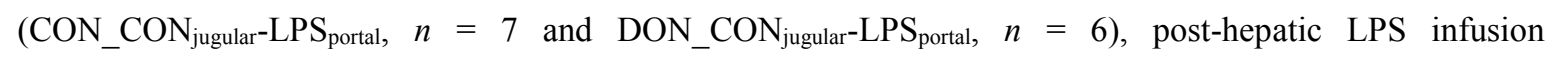

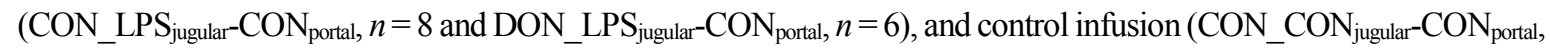
$n=7$ and DON_CON jugular $\left._{-}-\mathrm{CON}_{\text {portal }}, n=7\right)$. Infusion from time 0 until 60 min with $7.5 \mu \mathrm{g} \mathrm{LPS} / \mathrm{kg} \mathrm{BW}$ in $0.9 \%$ saline. Feed was offered during 15 min prior to infusion start. Blood samples were collected at times: $-30,15$, $30,45,60,75,90,120,150,180 \mathrm{~min}$. 

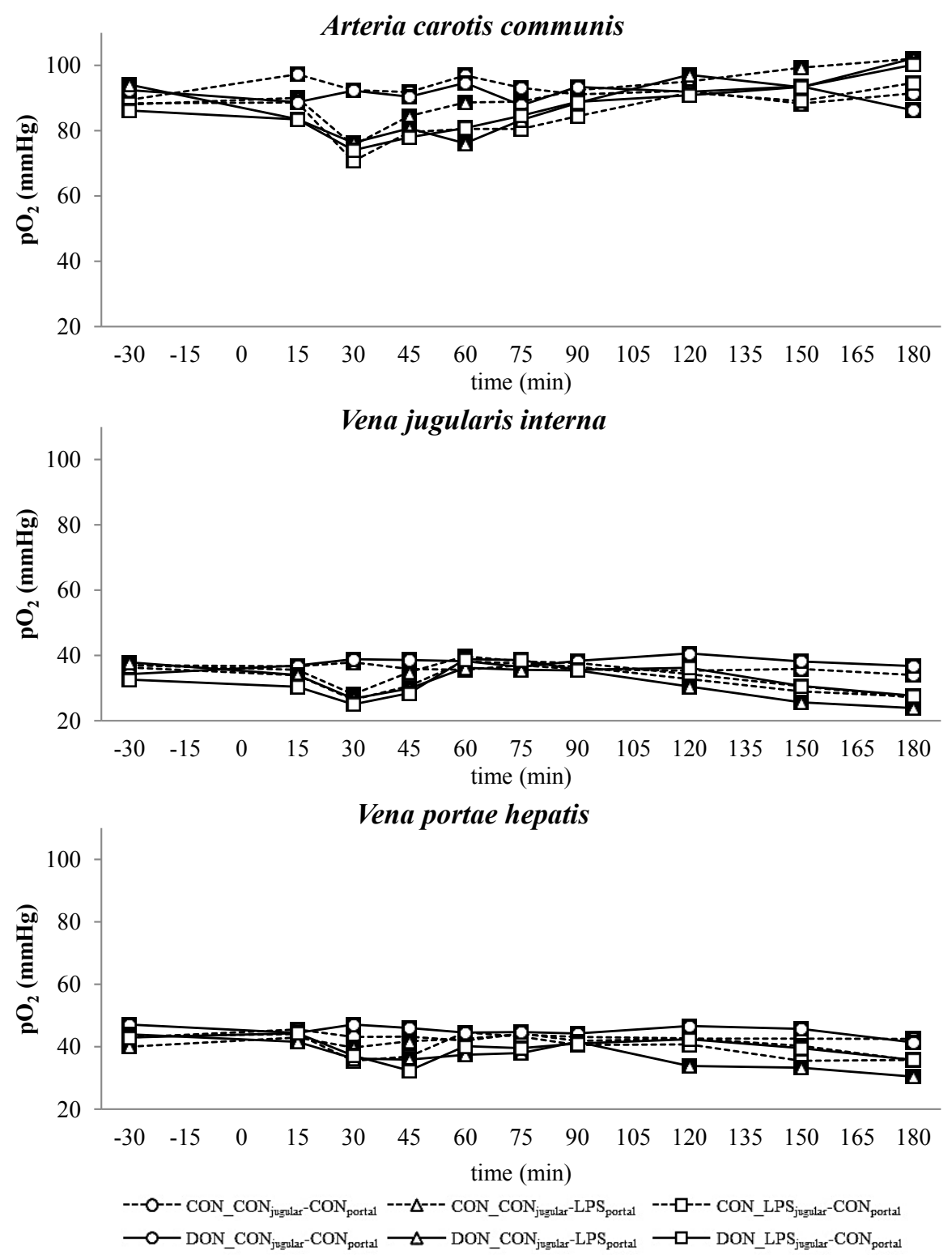

Figure 1. Effect of chronic enteral Fusarium toxin deoxynivalenol (DON) exposure and pre- or post-hepatic E. coli lipopolysaccharide (LPS) infusion on arterial, jugular or portal blood partial oxygen pressure $\left(\mathrm{OO}_{2}\right)$ in pigs. Reference value: $98 \mathrm{mmHg}$ in arterial blood [26]. Barrows were either fed a DON contaminated ration $(4.59 \mathrm{mg} / \mathrm{kg}$ feed $)$ or control feed during 29 days. Infusion groups were divided as follows: pre-hepatic LPS infusion $\left(C O N \_C O N\right.$ jugular-LPS $S_{\text {portal }}, n=7$ and DON_CON jugular-LPS $\left._{\text {portal }}, n=6\right)$, post-hepatic LPS infusion (CON_LPS jugular-CON portal $_{1}, n=8$ and DON_LPS jugular-CON $_{\text {portal }}, n=6$ ), and control infusion (CON_CON jugular $_{\text {-CON }}$ portal, $n=7$ and DON_CON ${ }_{\text {jugular }}-\mathrm{CON}_{\text {portal }}, n=7$ ). Infusion from time 0 until $60 \mathrm{~min}$ with $7.5 \mu \mathrm{g}$ LPS $/ \mathrm{kg}$ BW in $0.9 \%$ saline. Feed was offered during 15 min prior to infusion start. LSMeans. PSEM $=2.89$. Significance: Group $(\mathrm{G}): p=0.003$; Catheter (C): $p \leq 0.001$; Time $(\mathrm{T}): p \leq 0.001 ; \mathrm{G} \times \mathrm{C} \times \mathrm{T}: p \leq 0.001$. 


\subsection{Blood Gas Analysis}

\subsubsection{Partial Oxygen Pressure}

Arterial, jugular and portal partial oxygen pressures $\left(\mathrm{pO}_{2}\right)$ are illustrated in Figure 1. On average, partial $\mathrm{O}_{2}$ pressures of the control group were $92.42 \mathrm{mmHg}$ in arterial, $36.07 \mathrm{mmHg}$ in jugular and $43.08 \mathrm{mmHg}$ in portal blood $(\mathrm{SEM}=1.21)$, respectively. The arterial $p \mathrm{O} 2$ was near the physiological reference value of $98 \mathrm{mmHg}$ [26]. Between 15 and $60 \mathrm{~min}$, a general decrease in $p \mathrm{O}_{2}$ in all LPS-infused groups (compared to their control groups) was observed at all infusion sites $(p<0.05)$, which started to return to base level at $60 \mathrm{~min}$. The decrease was most pronounced in arterial blood. Thereafter, a subsequent increase in $p \mathrm{O}_{2}$ in all LPS-infused groups until 180 min was observed $(p<0.05)$. At jugular and portal sampling sites, $p \mathrm{O}_{2}$ decreased after 90 min, again below the control group level, and decreased thereafter until $120 \mathrm{~min}$ in jugular $(p<0.05)$ and $180 \mathrm{~min}$ in portal $(p<0.05)$ blood. A significant effect of DON exposure on arterial $p \mathrm{O}_{2}$ was observed at $60 \mathrm{~min}$ with pre-hepatic LPS-infused control-fed pigs starting to return to base level earlier than their DON-fed counter parts $(p<0.01)$. No effect of DON treatment was observed in post-hepatic infused animals. No DON effects were observed on jugular and portal $p \mathrm{O}_{2}$. Portal $p \mathrm{O}_{2}$ pressures were subjected more to fluctuations compared to arterial and portal $p \mathrm{O}_{2}$.

\subsubsection{Partial Carbon Dioxide Pressure}

Partial $\mathrm{CO}_{2}$ pressure $\left(\mathrm{pCO}_{2}\right)$ of control group was $37.80 \mathrm{mmHg}$ in arterial, $47.59 \mathrm{mmHg}$ in jugular and $52.42 \mathrm{mmHg}$ in portal blood $(\mathrm{SEM}=0.75)$ on average. At the arterial and jugular sampling site, a $\mathrm{pCO}_{2}$ below the physiological reference range $(50 \mathrm{mmHg})$ [26] was observed during the entire course of the trial and in all groups. Only arterial $\mathrm{pCO}_{2}$ was influenced by LPS treatment (Figure 2). From 120-180 min, a steady decrease was observed compared to the control group $(p<0.05)$. A slight portal $\mathrm{pCO}_{2}$ increase was observed from $-30 \mathrm{~min}$ until $180 \mathrm{~min}$ in all groups (Figure 2). Chronic oral exposure to DON had no impact on $\mathrm{pCO}_{2}$ irrespective of LPS infusion site. In jugular, as well as portal blood samples, undirected fluctuations were observed. 

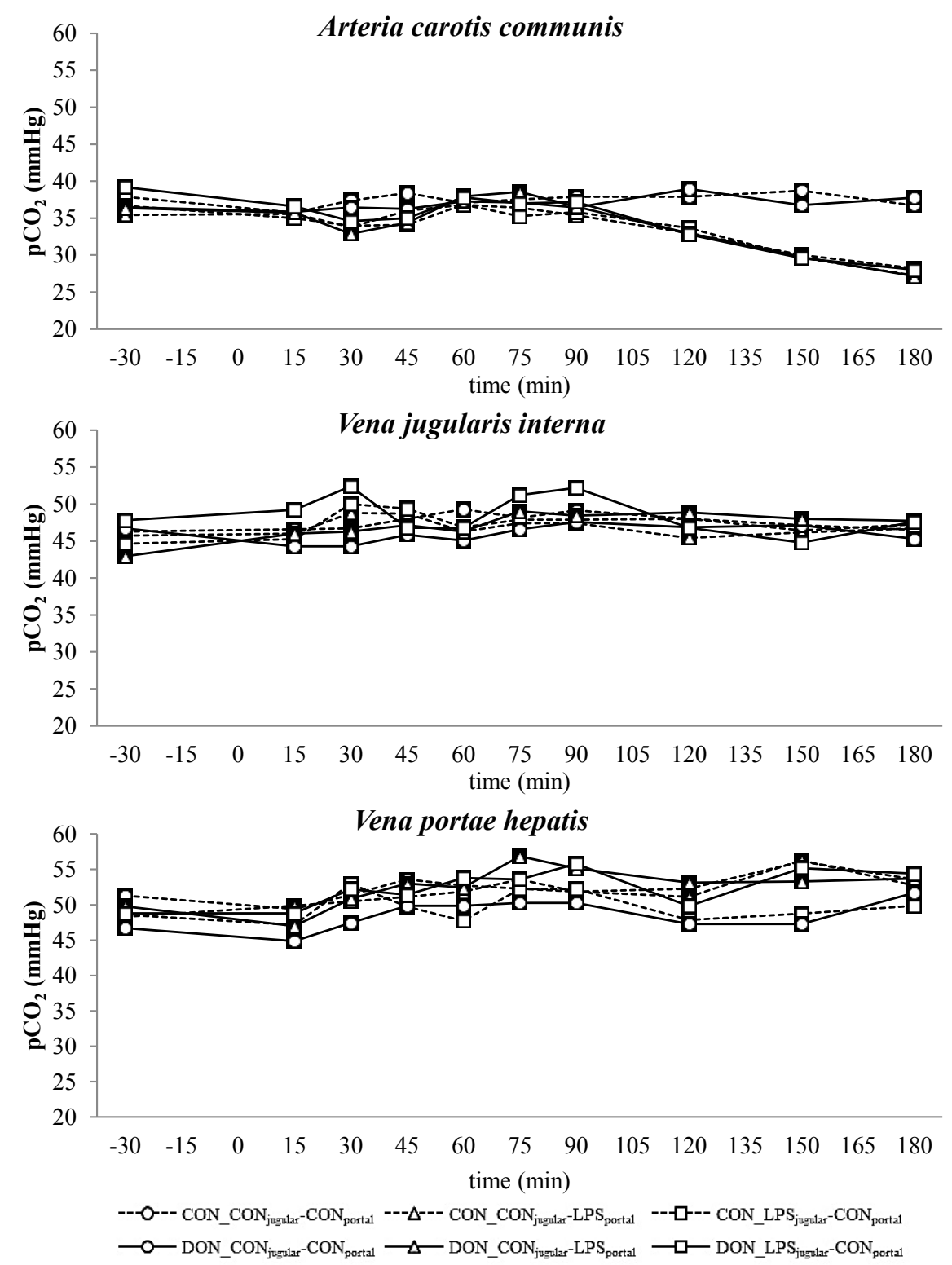

Figure 2. Effect of chronic enteral Fusarium toxin deoxynivalenol (DON) exposure and pre- or post-hepatic E. coli lipopolysaccharide (LPS) infusion on arterial, jugular or portal blood partial carbon dioxide pressure $\left(p \mathrm{CO}_{2}\right)$ in pigs. Reference value: $50 \mathrm{mmHg}$ in arterial blood [26]. Barrows were either fed a DON contaminated ration $(4.59 \mathrm{mg} / \mathrm{kg}$ feed $)$ or control feed during 29 days. Infusion groups were divided as follows: pre-hepatic LPS infusion (CON_CON jugular-LPS $_{\text {portal, }} n=7$ and DON_CON jugular-LPS $_{\text {portal }}, n=6$ ), post-hepatic LPS infusion (CON_LPS jugular-CON portal, $n=8$ and DON_LPS jugular-CON $_{\text {portal }}, n=6$ ), and control infusion (CON_CON jugular $_{\text {-CON }}$ portal, $n=7$ and DON_CON ${ }_{\text {jugular }}-\mathrm{CON}_{\text {portal }}, n=7$ ). Infusion from time 0 until 60 min with $7.5 \mu \mathrm{g}$ LPS $/ \mathrm{kg} \mathrm{BW}$ in $0.9 \%$ saline. Feed was offered during 15 min prior to infusion start. LSMeans. PSEM $=1.75$. Significance: Group $(\mathrm{G}): p=0.28$; Catheter (C): $p \leq 0.001$; Time (T): $p \leq 0.001 ; \mathrm{G} \times \mathrm{C} \times \mathrm{T}: p \leq 0.001$. 


\subsection{Electrolytes}

No significant effects of DON and LPS were observed on electrolytes $\left(\mathrm{Na}^{+}, \mathrm{Cl}^{-}, \mathrm{K}^{+}, i \mathrm{Ca}^{2+}\right)$. However, catheter site and collecting time significantly influenced electrolyte concentrations (Table 2). Slight fluctuations were observed for $\mathrm{Na}^{+}, \mathrm{K}^{+}$and $i \mathrm{Ca}^{2+}$ concentrations at different times, combined with minimal variation of the data, contributing to significant time and group $\times$ catheter $\times$ time effects. A significant catheter site effect was detected due to the generally higher jugular concentrations compared to arterial and portal levels at all groups. Electrolytes did not deviate from their respective physiological values [26,27] (Table 2).

Table 2. Effect of chronic enteral deoxynivalenol (DON) exposure and pre- or post-hepatic E. coli lipopolysaccharide (LPS) infusion on arterial, venous or portal blood electrolytes in pigs.

\begin{tabular}{ccccccc}
\hline \multirow{2}{*}{ Parameter } & \multirow{2}{*}{$\begin{array}{c}\text { Overall } \\
\text { Mean }\end{array}$} & PSEM & \multicolumn{5}{c}{$\boldsymbol{p}$-Values } \\
\cline { 4 - 7 } & 142.90 & 1.15 & 0.449 & $\mathbf{< 0 . 0 0 1}$ & $<\mathbf{0 . 0 0 1}$ & 0.629 \\
$\mathrm{Na}^{+}(\mathrm{mmol} / \mathrm{L})$ & 105.45 & 1.16 & 0.702 & $<\mathbf{0 . 0 0 1}$ & $<\mathbf{0 . 0 0 1}$ & 0.67 \\
$\mathrm{Cl}^{-}(\mathrm{mmol} / \mathrm{L})$ & 4.28 & 0.16 & 0.585 & $<\mathbf{0 . 0 0 1}$ & $<\mathbf{0 . 0 0 1}$ & $<\mathbf{0 . 0 0 1}$ \\
$\mathrm{K}^{+}(\mathrm{mmol} / \mathrm{L})$ & 1.37 & 0.03 & 0.985 & $<\mathbf{0 . 0 0 1}$ & $<\mathbf{0 . 0 0 1}$ & $\mathbf{0 . 0 0 6}$ \\
$i \mathrm{Ca}^{2+}(\mathrm{mmol} / \mathrm{L})$ & &
\end{tabular}

Notes: $\mathrm{Na}^{+}=$sodium (reference: $140-160 \mathrm{mmol} / \mathrm{L}^{1}$ ); $\mathrm{Cl}^{-}=$chloride (reference: $102-106 \mathrm{mmol} / \mathrm{L}^{1}$ ); $\mathrm{K}^{+}=$ potassium (reference: $4.0-5.0 \mathrm{mmol} / \mathrm{L}^{1}$ ); $i \mathrm{Ca}^{2+}=$ calcium iones (reference: $0.87-1.45 \mathrm{mmol} / \mathrm{L}^{2}$ ); ${ }^{1}=[26] ;{ }^{2}=[27]$; references in venous blood; ${ }^{\mathrm{T}}=$ trend $(p \leq 0.10) ; \mathrm{PSEM}=$ pooled standard error of Means. Barrows were either fed a DON contaminated ration (DON; $4.59 \mathrm{mg} / \mathrm{kg}$ feed) or control feed (CON) during 29 days. Infusion groups

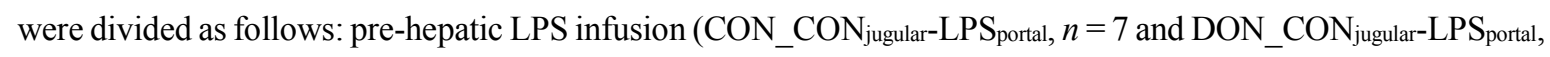
$n=6$ ), post-hepatic LPS infusion (CON_LPS jugular-CON ${ }_{\text {portal }}, n=8$ and DON_LPS jugular-CON $_{\text {portal }}, n=6$ ), and control infusion (CON_CON jugular-CON $\mathrm{Cortal}_{1}, n=7$ and DON_CON $\mathrm{jugular}_{-} \mathrm{CON}_{\text {portal }}, n=7$ ). Infusion from time 0 until $60 \mathrm{~min}$ with $7.5 \mu \mathrm{g} \mathrm{LPS} / \mathrm{kg} \mathrm{BW}$ in $0.9 \%$ saline. Feed was offered during $15 \mathrm{~min}$ prior to infusion start. Blood samples were collected at times: $-30,15,30,45,60,75,90,120,150,180 \mathrm{~min}$.

\subsection{Glucose}

A post-prandial increase in glucose until 15-30 min and a subsequent decrease until time 120 min to the base level was observed in all groups (Figure 3), most pronounced at the portal sampling site. The control (CON)-fed animals generally exhibited higher glucose levels at the portal sampling site at 30-45 min (depending on group) than DON-fed animals (Figure 3). The control group (CON_CON ${ }_{\text {jugular- }} \mathrm{CON}_{\text {portal }}$ ) maintained elevated post-prandial glucose levels for nearly the entire time course (significantly higher than other groups at $60 \mathrm{~min}, 90 \mathrm{~min}$ and $120 \mathrm{~min} ; p<0.05$ at $120 \mathrm{~min}$ ). 

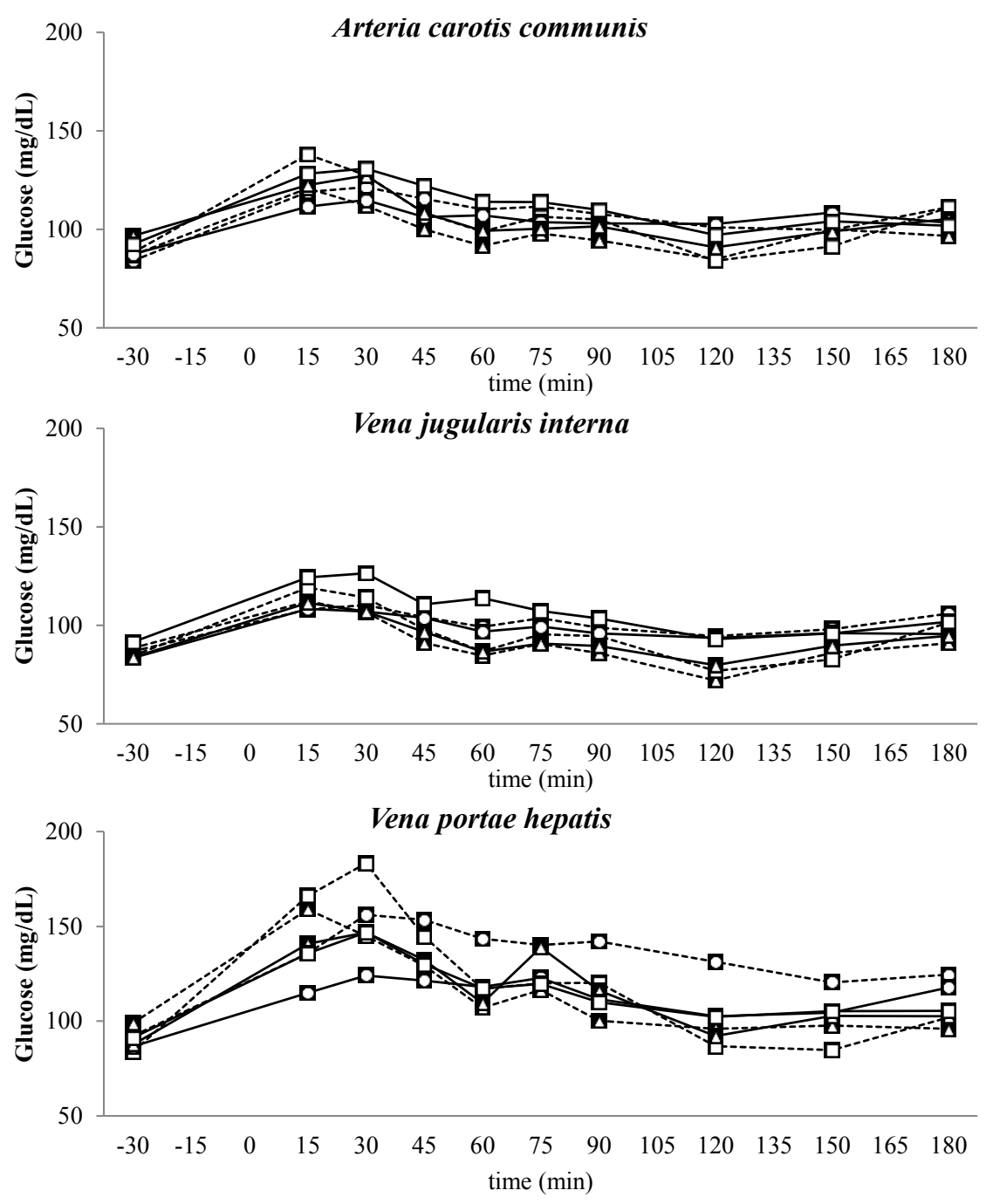

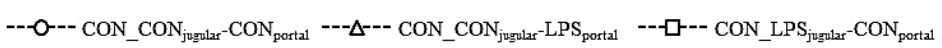

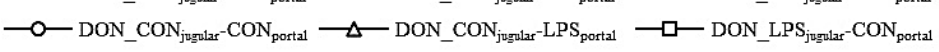

\begin{tabular}{ccccccccccc}
\hline \multicolumn{10}{c}{ post hoc test (p-value) CON_CON-CON vs. DON_CON-CON } \\
\hline time (min) & -30 & 15 & 30 & 45 & 60 & 75 & 90 & 120 & 150 & 180 \\
A. carotis & 0.819 & 0.599 & 0.652 & 0.518 & 0.833 & 0.587 & 0.745 & 0.912 & 0.546 & 0.587 \\
V.jugularis & 0.812 & 0.991 & 0.811 & 0.983 & 0.865 & 0.756 & 0.836 & 0.950 & 0.885 & 0.465 \\
V.portae & 0.686 & 0.131 & 0.024 & 0.021 & $0.066^{\mathrm{T}}$ & 0.207 & 0.028 & 0.043 & 0.247 & 0.632 \\
\hline
\end{tabular}

Figure 3. Effect of chronic enteral Fusarium toxin deoxynivalenol (DON) exposure and pre- or post-hepatic E. coli lipopolysaccharide (LPS) infusion on arterial, jugular or portal blood glucose in pigs. Reference value: $70-115 \mathrm{mg} / \mathrm{dL}$ in venous blood [26]. Barrows were either fed a DON contaminated ration $(4.59 \mathrm{mg} / \mathrm{kg}$ feed) or control feed during 29 days. Infusion groups were divided as follows: pre-hepatic LPS infusion (CON_CON jugular $_{\text {LPS }}$ portal,

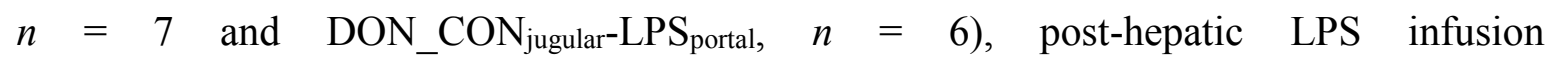
$\left(\mathrm{CON} \_\mathrm{LPS}_{\text {jugular- }}-\mathrm{CON}_{\text {portal }}, n=8\right.$ and DON_LPS jugular $_{\text {-CON }}$ portal, $\left.n=6\right)$, and control infusion (CON_CON jugular-CON $_{\text {portal }}, n=7$ and DON_CON $\mathrm{Nugular}_{-} \mathrm{CON}_{\text {portal }}, n=7$ ). Infusion from time 0 until $60 \mathrm{~min}$ with $7.5 \mu \mathrm{g} \mathrm{LPS} / \mathrm{kg} \mathrm{BW}$ in $0.9 \%$ saline. Feed was offered during $15 \mathrm{~min}$ prior to infusion start. LSMeans. PSEM $=1.75$. Significance: Group $(\mathrm{G}): p=0.28$; Catheter (C): $p \leq 0.001$; Time (T): $p \leq 0.001 ; \mathrm{G} \times \mathrm{C} \times \mathrm{T}: p \leq 0.001$. Table illustrates differences between DON- and CON-fed control-infused groups at different times. ${ }^{\mathrm{T}}=$ trend $(p \leq 0.10)$. 

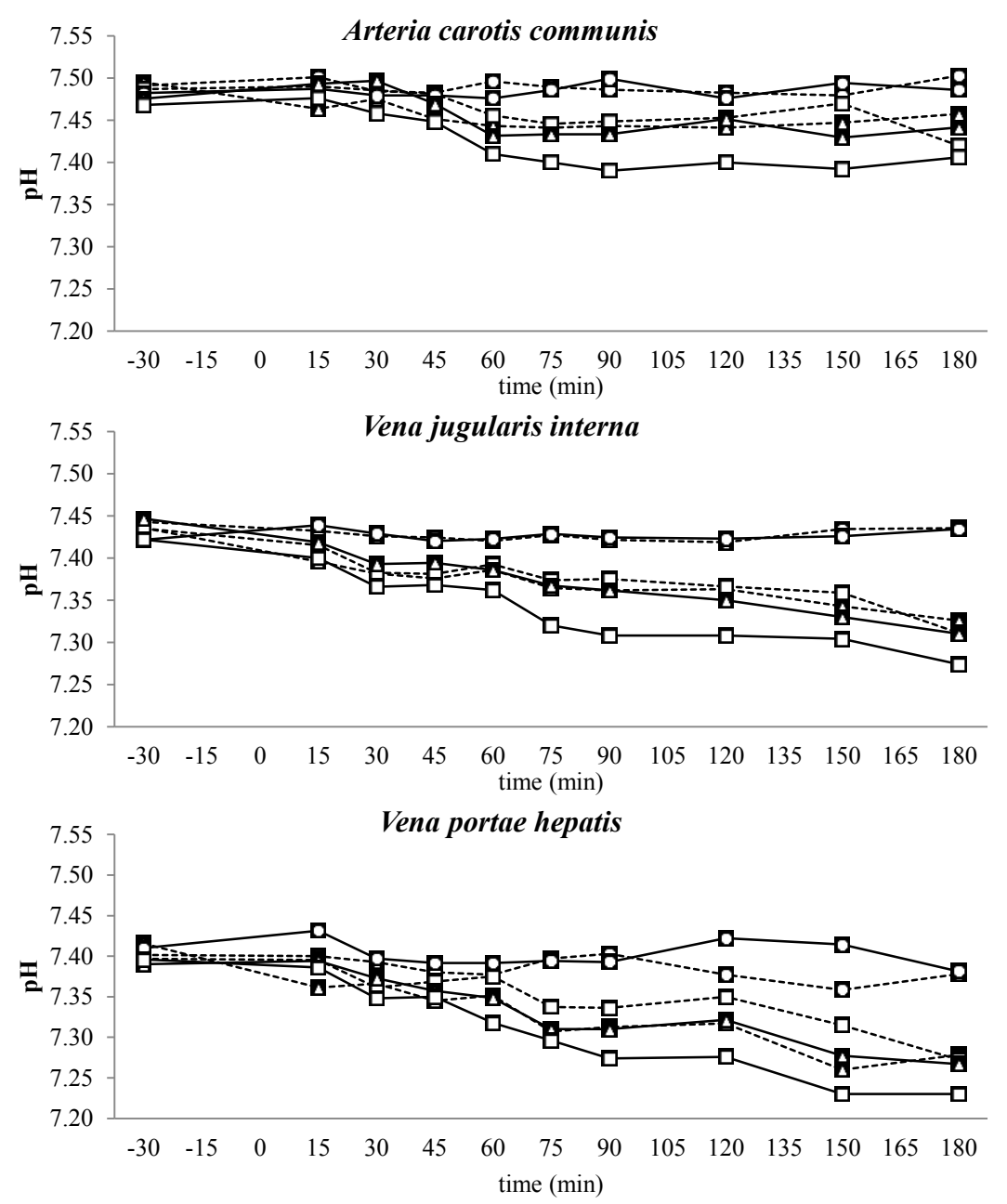

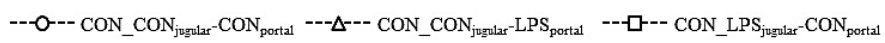

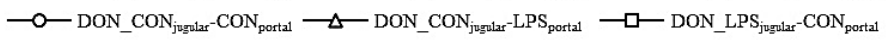

\begin{tabular}{ccccccccccc}
\hline \multicolumn{10}{c}{ post hoc test (p-value) CON_LPS-CON vs. DON_LPS-CON } \\
\hline time (min) & -30 & 15 & 30 & 45 & 60 & 75 & 90 & 120 & 150 & 180 \\
A. carotis & 0.499 & 0.611 & 0.327 & 0.236 & 0.105 & 0.105 & 0.038 & $0.061^{\mathrm{T}}$ & 0.006 & 0.623 \\
V.jugularis & 0.636 & 0.585 & 0.548 & 0.630 & 0.268 & $0.051^{\mathrm{T}}$ & 0.015 & 0.035 & 0.047 & 0.176 \\
V.portae & 0.975 & 0.736 & 0.637 & 0.495 & 0.039 & 0.132 & 0.024 & 0.007 & 0.002 & 0.129 \\
\hline
\end{tabular}

Figure 4. Effect of chronic enteral Fusarium toxin deoxynivalenol (DON) exposure and pre- or post-hepatic E. coli lipopolysaccharide (LPS) infusion on arterial, jugular or portal blood pH in pigs. Reference value: 7.42 in arterial blood [26]. Barrows were either fed a DON contaminated ration $(4.59 \mathrm{mg} / \mathrm{kg}$ feed) or control feed during 29 days. Infusion groups were divided as follows: pre-hepatic LPS infusion (CON_CON jugular-LPS portal $_{2} n=7$ and DON_CON jugular-LPS $_{\text {portal, }} n=6$ ), post-hepatic LPS infusion (CON_LPS jugular-CON portal, $_{\text {, }}$ $n=8$ and DON_LPS jugular-CON $\left._{\text {portal }}, n=6\right)$, and control infusion $\left(\mathrm{CON}_{-} \mathrm{CON}_{\text {jugular- }} \mathrm{CON}_{\text {portal }}\right.$, $n=7$ and DON_CON jugular-CON $\mathrm{COrtal}_{\text {, }} n=7$ ). Infusion from time 0 until 60 min with $7.5 \mu \mathrm{g} \mathrm{LPS} / \mathrm{kg} \mathrm{BW}$ in $0.9 \%$ saline. Feed was offered during $15 \mathrm{~min}$ prior to infusion start. LSMeans. PSEM = 0.02. Significance: Group (G): $p \leq 0.001$; Catheter $(\mathrm{C}): p \leq 0.001$; Time $(\mathrm{T}): p \leq 0.001 ; \mathrm{G} \times \mathrm{C} \times \mathrm{T}: p \leq 0.001$. Table illustrates differences between DON and CON fed post-hepatic LPS infused groups at different times. ${ }^{\mathrm{T}}=$ trend $(p \leq 0.10)$. 

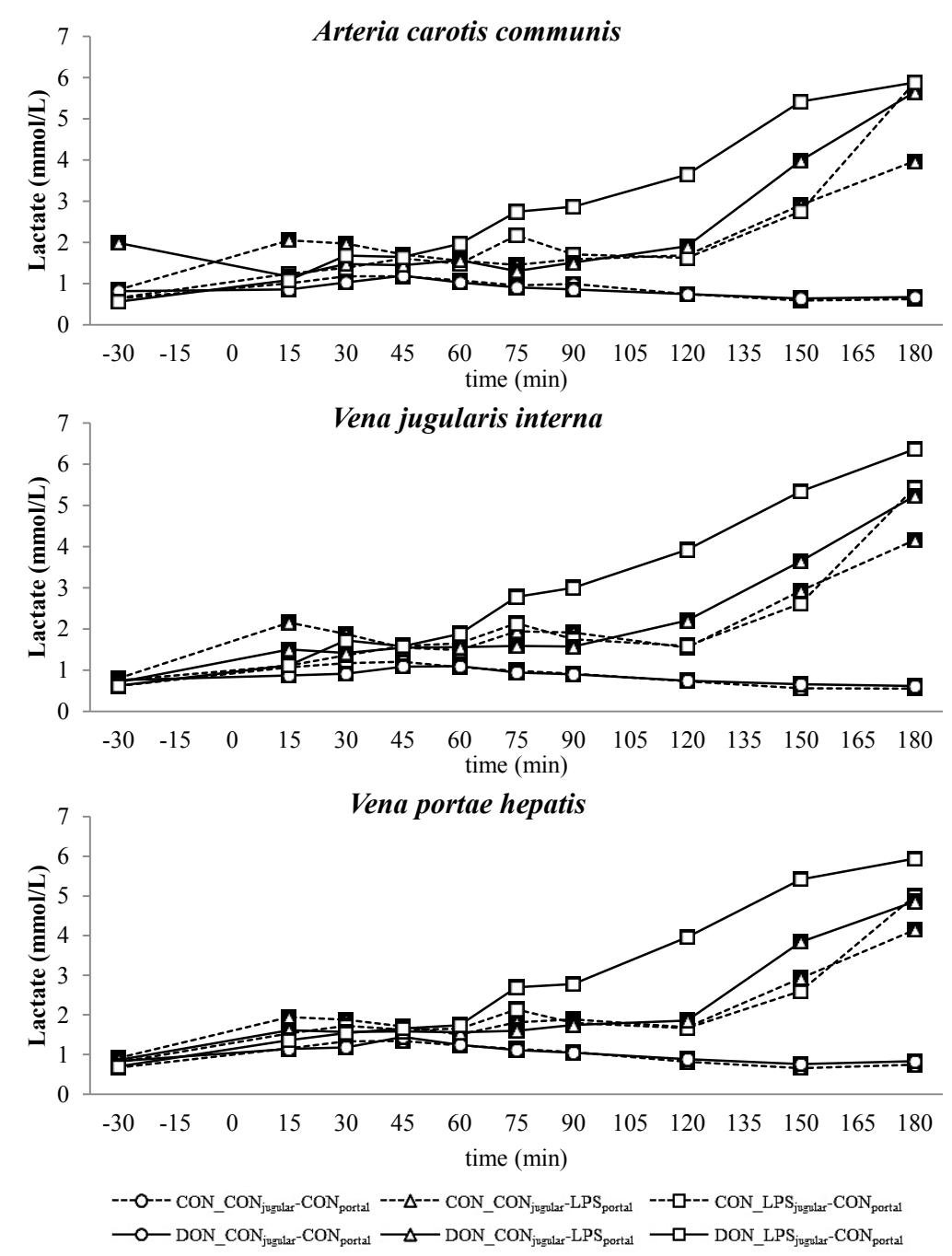

\begin{tabular}{|c|c|c|c|c|c|c|c|c|c|c|}
\hline \multicolumn{11}{|c|}{ post hoc test (p-value) CON_LPS-CON vs. DON_LPS-CON } \\
\hline time (min) & -30 & 15 & 30 & 45 & 60 & 75 & 90 & 120 & 150 & 180 \\
\hline A. carotis & 0.912 & 0.845 & 0.714 & 0.969 & 0.558 & 0.484 & 0.161 & 0.014 & 0.001 & 0.999 \\
\hline V. jugularis & 0.871 & 0.993 & 0.656 & 0.993 & 0.775 & 0.424 & 0.120 & 0.004 & 0.001 & 0.245 \\
\hline$V$ portae & 0.904 & 0.838 & 0.824 & 0.965 & 0.911 & 0.484 & 0.223 & 0.004 & $<0.001$ & 0.252 \\
\hline
\end{tabular}

Figure 5. Effect of chronic enteral Fusarium toxin deoxynivalenol (DON) exposure and pre- or post-hepatic E. coli lipopolysaccharide (LPS) infusion on arterial, jugular or portal blood lactate in pigs. Reference: $0.84 \pm 0.24 \mathrm{mmol} / \mathrm{L}$ in venous blood [28]. Barrows were either fed a DON contaminated ration $(4.59 \mathrm{mg} / \mathrm{kg}$ feed) or control feed during 29 days. Infusion groups were divided as follows: pre-hepatic LPS infusion (CON_CON jugular $_{-} \mathrm{LPS}_{\text {portal }}, n=7$ and DON_CON jugular-LPS $\left._{\text {portal }}, n=6\right)$, post-hepatic LPS infusion (CON_LPS jugular-CON $\mathrm{N}_{\text {portal }}$, $n=8$ and DON_LPS jugular-CON $\left._{\text {portal }}, n=6\right)$, and control infusion $\left(\mathrm{CON}_{-} \mathrm{CON}_{\text {jugular }}-\mathrm{CON}_{\text {portal }}\right.$, $n=7$ and DON_CON jugular-CON $\left._{\text {portal, }} n=7\right)$. Infusion from time 0 until 60 min with $7.5 \mu \mathrm{g} \mathrm{LPS} / \mathrm{kg} \mathrm{BW}$ in $0.9 \%$ saline. Feed was offered during $15 \mathrm{~min}$ prior to infusion start. LSMeans. PSEM = 0.56. Significance: Group $(\mathrm{G}): p \leq 0.001$; Catheter $(\mathrm{C}): p=0.78$; Time (T): $p \leq 0.001 ; \mathrm{G} \times \mathrm{C} \times \mathrm{T}: p \leq 0.001$. Table illustrates differences between DON and CON fed post-hepatic LPS infused groups at different times. ${ }^{\mathrm{T}}=$ trend $(p \leq 0.10)$. 

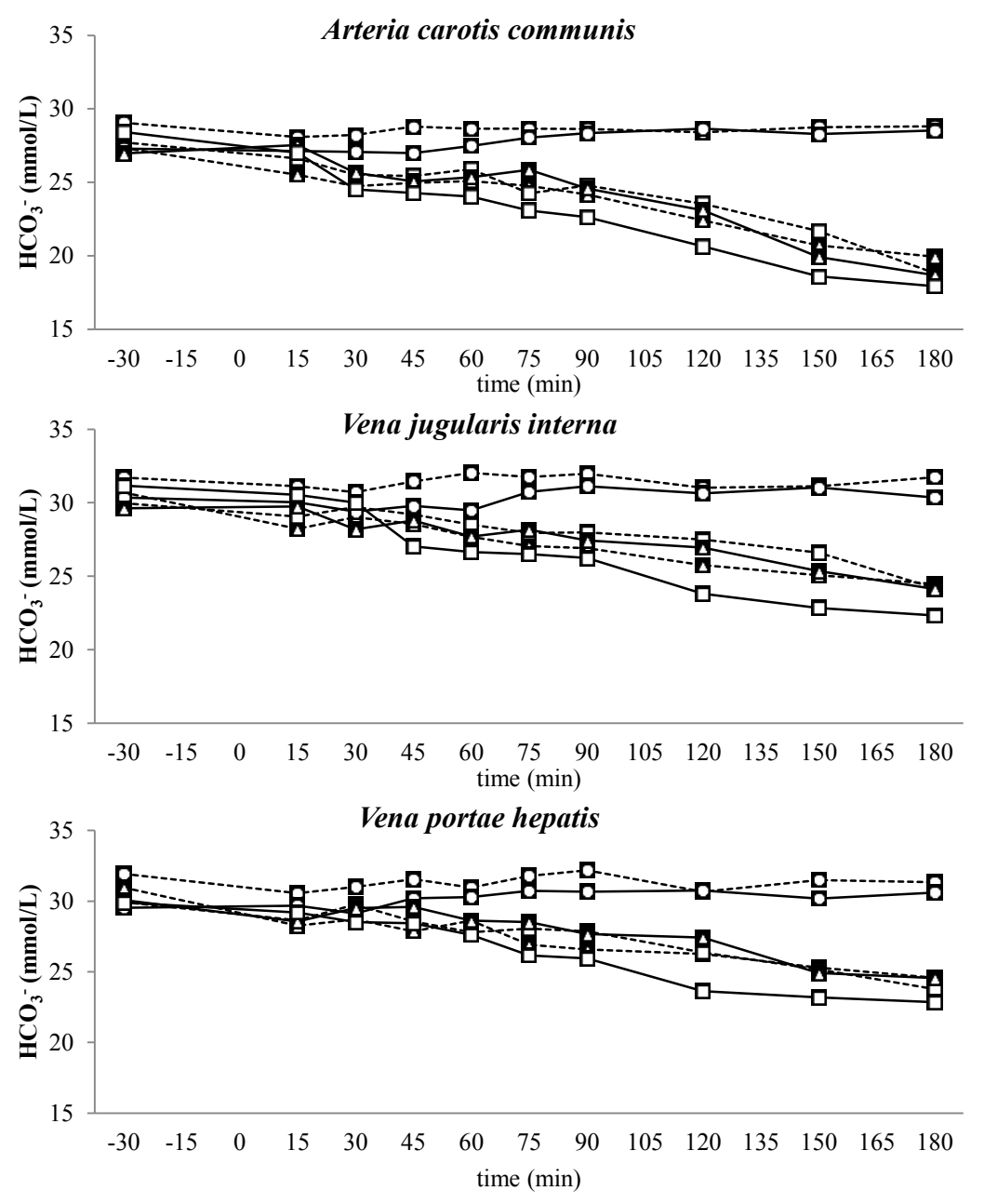

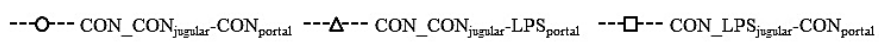

$\longrightarrow$ - DON_CON ${ }_{\text {jugular }}-\mathrm{CON}_{\text {portal }} \rightarrow \Delta-$ DON_CON $_{\text {jugular }}-\mathrm{LPS}_{\text {portal }} \rightarrow \square-$ DON_LPS $_{\text {jugular }}-\mathrm{CON}_{\text {portal }}$

\begin{tabular}{|c|c|c|c|c|c|c|c|c|c|c|}
\hline \multicolumn{11}{|c|}{ post hoc test (p-value) CON_LPS-CON vs. DON_LPS-CON } \\
\hline time (min) & -30 & 15 & 30 & 45 & 60 & 75 & 90 & 120 & 150 & 180 \\
\hline A. carotis & 0.639 & 0.789 & 0.506 & 0.443 & 0.215 & 0.431 & 0.153 & $0.054^{\mathrm{T}}$ & 0.042 & 0.558 \\
\hline V. jugularis & 0.423 & 0.314 & 0.835 & 0.145 & 0.206 & 0.327 & 0.238 & 0.014 & 0.012 & 0.205 \\
\hline$V \cdot$ portae & 0.980 & 0.714 & 0.408 & 0.934 & 0.896 & 0.202 & 0.195 & $0.066^{\mathrm{T}}$ & 0.181 & 0.539 \\
\hline
\end{tabular}

Figure 6. Effect of chronic enteral Fusarium toxin deoxynivalenol (DON) exposure and pre- or post-hepatic E. coli lipopolysaccharide (LPS) infusion on arterial, jugular or portal blood bicarbonate $\left(\mathrm{HCO}_{3}{ }^{-}\right)$in pigs. Reference range: $20-30 \mathrm{mmol} / \mathrm{L}$ in arterial blood [26]. Barrows were either fed a DON contaminated ration $(4.59 \mathrm{mg} / \mathrm{kg}$ feed) or control feed during 29 days. Infusion groups were divided as follows: pre-hepatic LPS infusion (CON_CON jugular-LPS $_{\text {portal }}, n=7$ and DON_CON jugular-LPS portal $_{\text {_ }} n=6$ ), post-hepatic LPS infusion (CON_LPS jugular-CON $_{\text {portal }}, n=8$ and DON_LPS jugular $_{-} \mathrm{CON}_{\text {portal }}, n=6$ ), and control infusion (CON_CON jugular- $_{\text {CON }}$ portal, $n=7$ and $\mathrm{DON}_{-} \mathrm{CON}_{\text {jugular- }} \mathrm{CON}_{\text {portal }}, n=7$ ). Infusion from time 0 until $60 \mathrm{~min}$ with $7.5 \mu \mathrm{g} \mathrm{LPS} / \mathrm{kg} \mathrm{BW}$ in $0.9 \%$ saline. Feed was offered during 15 min prior to infusion start. LSMeans. PSEM $=1.18$. Significance: Group $(\mathrm{G}): p \leq 0.001$; Catheter (C): $p \leq 0.001$; Time (T): $p \leq 0.001 ; \mathrm{G} \times \mathrm{C} \times \mathrm{T}: p \leq 0.001$. Table illustrates differences between DON and CON fed post-hepatic LPS infused groups at different times, $\mathrm{T}=$ trend $(p \leq 0.10)$. 

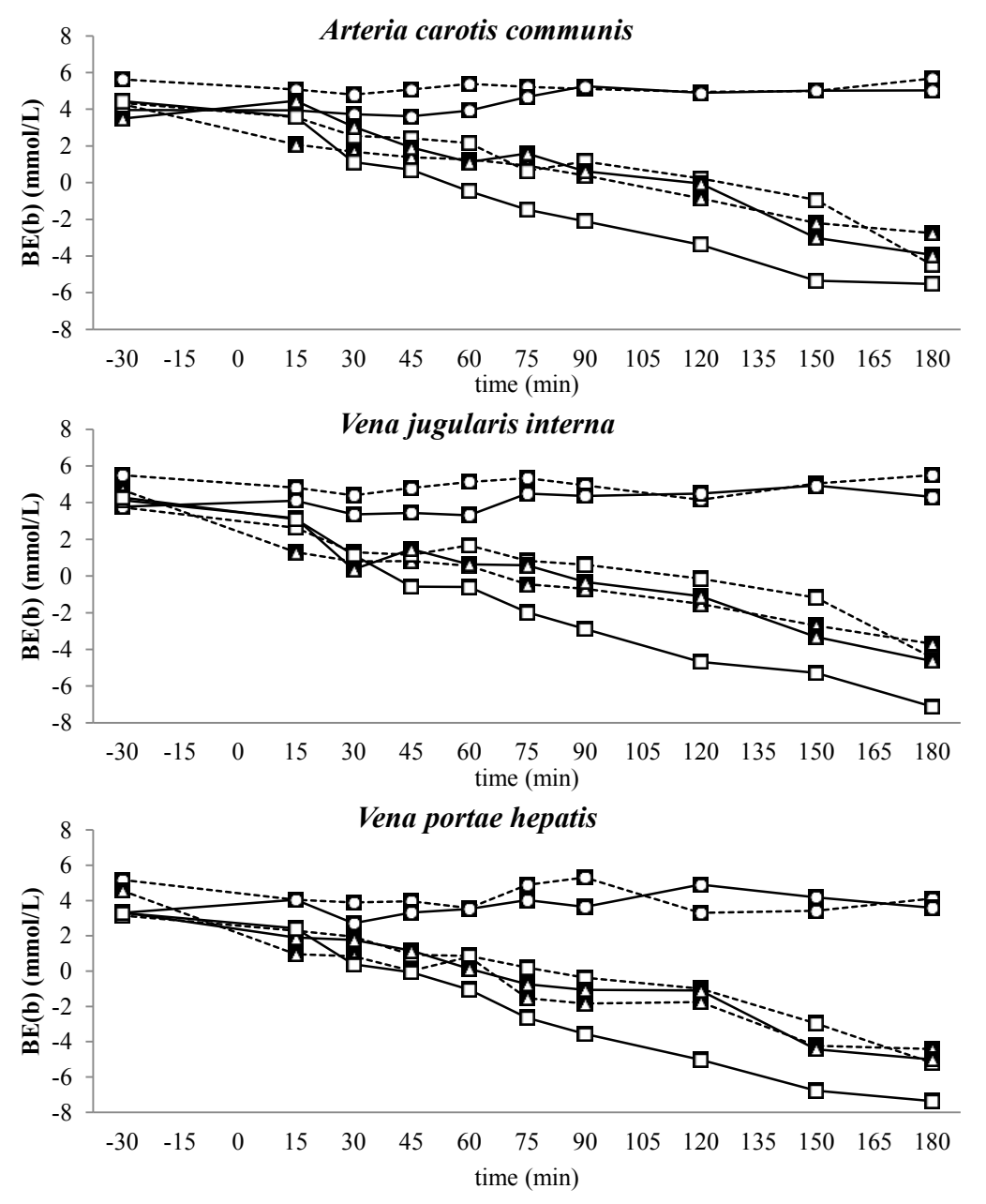

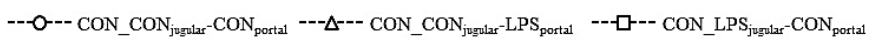

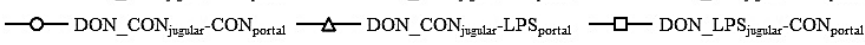

\begin{tabular}{cccccccccccc}
\hline \multicolumn{10}{c}{ post hoc test (p-value) CON_LPS-CON vs. DON_LPS-CON } \\
\hline time (min) & -30 & 15 & 30 & 45 & 60 & 75 & 90 & 120 & 150 & 180 \\
A.carotis & 0.957 & 0.988 & 0.413 & 0.324 & 0.133 & 0.226 & $0.062^{\mathrm{T}}$ & 0.039 & 0.012 & 0.545 \\
V.jugularis & 0.757 & 0.787 & 0.935 & 0.310 & 0.186 & 0.103 & 0.042 & 0.009 & 0.018 & 0.119 \\
V.portae & 0.931 & 0.941 & 0.364 & 0.566 & 0.268 & $0.099^{\mathrm{T}}$ & $0.064^{\mathrm{T}}$ & 0.020 & 0.027 & 0.214 \\
\hline
\end{tabular}

Figure 7. Effect of chronic enteral Fusarium toxin deoxynivalenol (DON) exposure and pre- or post-hepatic E. coli lipopolysaccharide (LPS) infusion on arterial, jugular or portal blood base-excess $(\mathrm{BE}(\mathrm{b}))$ in pigs. Reference range: $-3.5-3.5 \mathrm{mmol} / \mathrm{L}$ in arterial blood [26]. Barrows were either fed a DON contaminated ration $(4.59 \mathrm{mg} / \mathrm{kg}$ feed) or control feed during 29 days. Infusion groups were divided as follows: pre-hepatic LPS infusion (CON_CON jugular-LPS $_{\text {portal }}, n=7$ and DON_CON jugular-LPS portal $_{\text {, }} n=6$ ), post-hepatic LPS infusion (CON_LPS jugular-CON portal $_{1}, n=8$ and DON_LPS jugular-CON $\mathrm{Cortal}_{1}, n=6$ ), and control infusion (CON_CON jugular-CON $_{\text {portal }}, n=7$ and $\mathrm{DON}_{-} \mathrm{CON}_{\text {jugular- }} \mathrm{CON}_{\text {portal }}, n=7$ ). Infusion from time 0 until $60 \mathrm{~min}$ with $7.5 \mu \mathrm{g} \mathrm{LPS} / \mathrm{kg} \mathrm{BW}$ in $0.9 \%$ saline. Feed was offered during 15 min prior to infusion start. LSMeans. PSEM $=1.18$. Significance: Group $(\mathrm{G}): p \leq 0.001$; Catheter (C): $p \leq 0.001$; Time (T): $p \leq 0.001 ; \mathrm{G} \times \mathrm{C} \times \mathrm{T}: p \leq 0.001$. Table illustrates differences between DON and CON fed post-hepatic LPS infused groups at different times $\mathrm{T}=$ trend $(p \leq 0.10)$. 

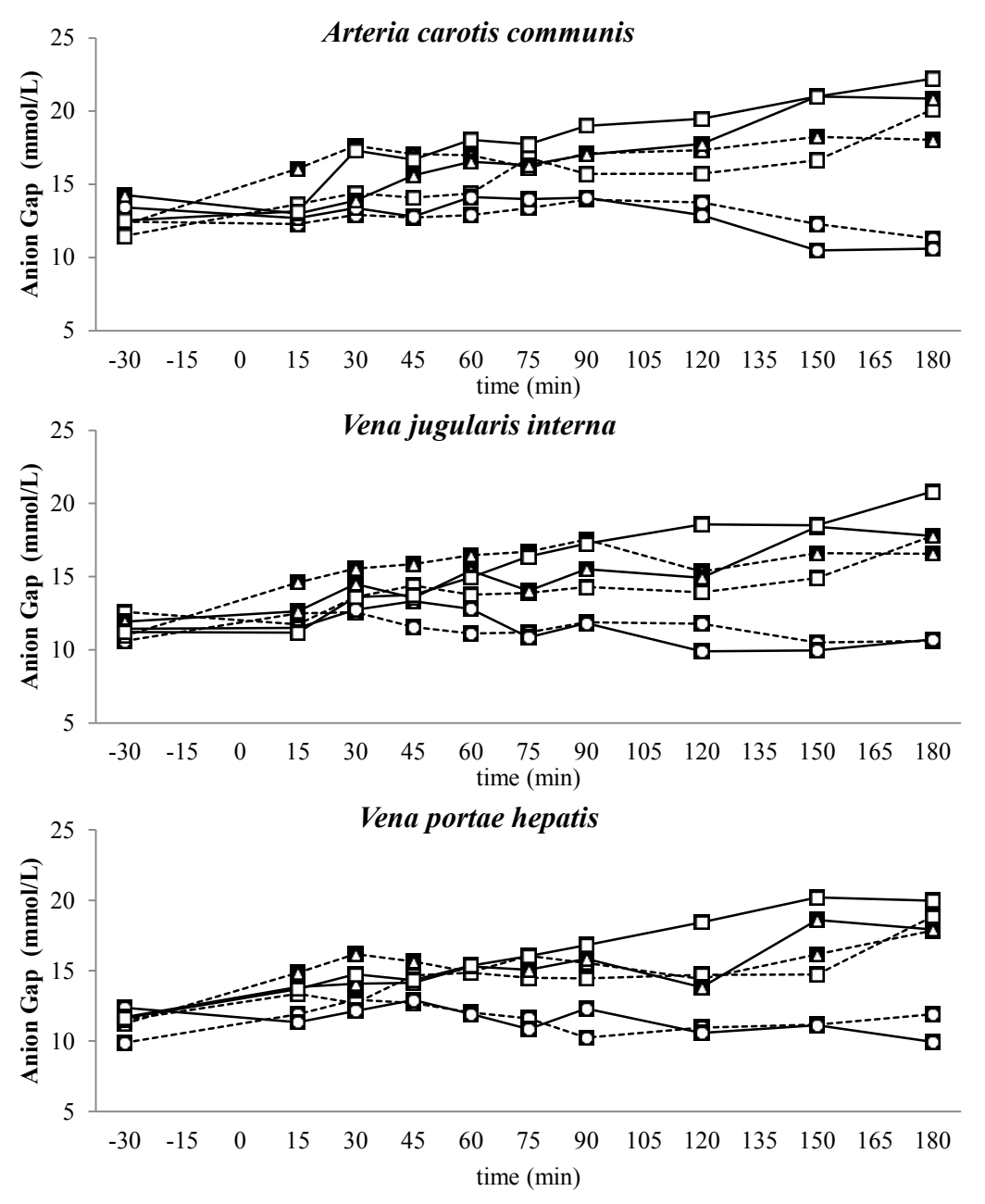

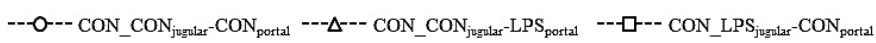

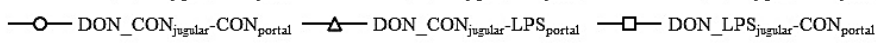

\begin{tabular}{cccccccccccc}
\hline \multicolumn{10}{c}{ post hoc test (p-value) CON_LPS-CON vs. DON_LPS-CON } \\
\hline time (min) & -30 & 15 & 30 & 45 & 60 & 75 & 90 & 120 & 150 & 180 \\
A.carotis & 0.564 & 0.792 & 0.115 & 0.162 & 0.048 & 0.612 & $0.075^{\mathrm{T}}$ & 0.043 & 0.019 & 0.253 \\
V.jugularis & 0.451 & 0.759 & 1.000 & 0.716 & 0.516 & 0.170 & 0.102 & 0.011 & 0.048 & $0.095^{\mathrm{T}}$ \\
V.portae & 0.951 & 0.855 & 0.268 & 0.850 & 0.784 & 0.392 & 0.192 & 0.041 & 0.003 & 0.535 \\
\hline
\end{tabular}

Figure 8. Effect of chronic enteral Fusarium toxin deoxynivalenol (DON) exposure and pre- or post-hepatic $E$. coli lipopolysaccharide (LPS) infusion on arterial, jugular or portal blood anion-gap Gap $\left(\mathrm{K}^{+}\right)$in pigs. Reference range: $10-25 \mathrm{mmol} / \mathrm{L}$ in venous blood [27]. Barrows were either fed a DON contaminated ration $(4.59 \mathrm{mg} / \mathrm{kg}$ feed) or control feed during 29 days. Infusion groups were divided as follows: pre-hepatic LPS infusion (CON_CON jugular-LPS $_{\text {portal }}, n=7$ and DON_CON jugular-LPS portal $_{\text {_ }} n=6$ ), post-hepatic LPS infusion (CON_LPS jugular-CON $_{\text {portal }}, n=8$ and DON_LPS jugular $\left._{-} \mathrm{CON}_{\text {portal }}, n=6\right)$, and control infusion (CON_CON jugular- $_{\text {CON }}$ portal, $n=7$ and $\mathrm{DON}_{-} \mathrm{CON}_{\text {jugular- }}-\mathrm{CON}_{\text {portal }}, n=7$ ). Infusion from time 0 until $60 \mathrm{~min}$ with $7.5 \mu \mathrm{g} \mathrm{LPS} / \mathrm{kg} \mathrm{BW}$ in $0.9 \%$ saline. Feed was offered during 15 min prior to infusion start. LSMeans. PSEM $=1.22$. Significance: Group $(\mathrm{G}): p \leq 0.001$; Catheter (C): $p \leq 0.001$; Time (T): $p \leq 0.001 ; \mathrm{G} \times \mathrm{C} \times \mathrm{T}: p \leq 0.001$. Table illustrates differences between DON and CON fed post-hepatic LPS infused groups at different times. ${ }^{\mathrm{T}}=$ trend $(p \leq 0.10)$. 


\subsection{Acid-Base Balance}

In all LPS-infused pigs, lactic acidosis was induced, and the acid-base balance variables were altered accordingly (Figures 4-8). Compared to the control group ( $\left.\mathrm{CON}_{-} \mathrm{CON}_{\text {jugular- }} \mathrm{CON}_{\text {portal }}\right)$, pH (Figure 4), bicarbonate (Figure 6) and base excess (BE; Figure 7) decreased and lactate (Figure 5) and anion-gap (AG; Figure 8) increased significantly $(p<0.05)$. The control group stayed in the physiological range [26] for $\mathrm{pH}$ (arterial), lactate (venous) [28], $\mathrm{HCO}_{3}{ }^{-}$(arterial) and $\mathrm{AG}$ (venous) [27], but $\mathrm{BE}$ (arterial) was above the reference range over the course of the trial with individual variations $(5.23 \mathrm{mmol} / \mathrm{L} \pm 0.79$; mean $\pm \mathrm{SE})$.

DON treatment had no effect on control and pre-hepatic LPS-infused groups, but a significant DON effect was observed in post-hepatic LPS-infused animals. Lactic acidosis was most pronounced in post-hepatic LPS-infused DON-fed pigs (DON_LPS jugular-CON $\mathrm{Nortal}_{\text {) }}$ at different times in comparison to their control-fed counterparts (CON_LPS jugular-CON $_{\text {portal }}$ ) and the pre-hepatic LPS-infused groups $(p<0.05)$ (shown in the post hoc tables, Figures $4-8)$. The DON_LPS jugular-CON $_{\text {portal }}$ group had a different time course compared to the other LPS groups for all variables of acid-base balance. This was most apparent in lactate, with a significant increase compared to the control already at $75-180 \mathrm{~min}$ at all catheters in contrast to the other LPS groups, which were only significantly different from the control at $150 \mathrm{~min}$ and $180 \mathrm{~min}$. Additionally, we observed a chronological sequence of lactic acidosis variables compared to the control: $\mathrm{pO}_{2}, \mathrm{HCO}_{3}{ }^{-}$and $\mathrm{BE}$ already changed at 30-45 min, followed by $\mathrm{pH}$ at $60 \mathrm{~min}$, and as the last variable, lactate rose significantly at $75 \mathrm{~min}$.

\section{Discussion}

In this study, LPS was used to induce an inflammatory response. All LPS-treated pigs exhibited typical clinical symptoms of an acute phase response [16,29,30], such as an increased respiratory rate, fever, tremor, cyanosis, followed by hyperemic conjunctivae, injected episcleral vessels or leukopenia (Tesch et al., 2015, submitted, [31]). In all groups at time -30 min and within the control group over the course of the trial, no significant alterations of metabolic and hematological variables were observed, and all parameters were within their physiological range. It therefore can be stated that the performed manipulations, such as the surgery and the sampling procedure, did not constitute confounding factors.

All LPS-treated animals exhibited a lactic acidosis as a consequence of LPS infusion [13]. Taking into consideration the observed $p \mathrm{O}_{2}, p \mathrm{CO}_{2}$ and lactate concentrations, we deduced that at first, acidosis was caused by a decreased $p \mathrm{O}_{2}$ (respiratory acidosis) and later on originated from an increase in systemic lactate concentration (metabolic acidosis) [32]. This is also mirrored in the variables BE and $\mathrm{AG}$, which are a reflection of the different variables that affect the acid-base balance [33]. The alterations observed in $\mathrm{AG}$ can solely be ascribed to changes in $\mathrm{HCO}_{3}{ }^{-}$concentrations, since other electrolytes $\left(\mathrm{Na}^{+}, \mathrm{K}^{+}, \mathrm{Cl}^{-}, \mathrm{Ca}^{2+}\right)$ were not influenced by the LPS challenge.

Since erythrocytes occupy a central role in oxygen transport, metabolism, as well as the acid-base balance, we also assessed red hemogram variables. No biologically-relevant influence of DON or LPS was observed on red hemogram variables at any time during the trial. This is in line with a study of Grenier et al. [34] in which the effects of the mycotoxins DON and fumonisin, alone or in combination with a subcutaneous ovalbumin injection on different hematology variables, were investigated. 
These results are further confirmed by two recent studies $[35,36]$ investigating the influence of low-dose ( $\leq 2 \mathrm{mg} / \mathrm{kg}$ feed) chronic oral DON exposure on red hemogram variables and electrolytes in piglets and pre-puberal gilts, respectively. However, in both studies, neither biologically-relevant alterations in red hemogram nor blood electrolytes were observed after four weeks of chronic oral DON exposure. Few studies have investigated the influence of an acute inflammatory response on sodium-potassium transport in red blood cells and skeletal muscle. Suri and colleagues [37] and Illner et al. [38] observed hyponatremia and hyperpotassemia and attributed this to alteration in the transport capacity of the RBC $\mathrm{Na}^{+} / \mathrm{K}^{+}$pump. This change in ion transport across RBC and skeletal muscles has also been observed in other studies $[39,40]$, but this hypothesis was challenged later on $[41,42]$. In our study, we did not detect any changes in blood electrolytes and, based on these previous articles, might speculate that the sodium-potassium transport in red blood cells and skeletal muscle after an LPS-challenge was not changed.

A decrease in systemic $p \mathrm{O}_{2}$ during the initial state of an acute inflammatory response has been documented before and is probably caused by a decreased cardiac output, as well as alterations in the respiratory rate and depth [43]. This assumption is supported by the concurrent observed increase in respiratory rate in LPS-infused animals during this trial (Tesch et al., 2015, submitted, [31]). After an initial decrease of arterial $p \mathrm{O}_{2}$, a continuous increase during the rest of the trial, accompanied by a decrease in jugular $p \mathrm{O}_{2}$ and no alterations in jugular $\mathrm{pCO}_{2}$, was observed. This observation is indicative of an increase in tissue oxygen consumption, rather than a decrease in overall oxygen availability. These findings are in line with previous studies showing that the hyperlactatemia observed during sepsis is most likely caused by alterations in the glycolytic pathway, rather than hypoxia, along with increased tissue oxygen consumption [44]. In human studies, Revelly and co-workers [45] described an increased glucose and lactate rate of appearance in the blood of septic patients compared to healthy subjects. Furthermore, the hyperlactatemia resulted from an increased endogenous lactate production in sepsis, whereas lactate clearance was not altered compared to healthy patients, confirming the impact of altered glycolytic pathways in the development of lactic acidosis. It is further assumed that during a state of acute inflammatory response, the rate of pyruvate formation exceeds the oxidative capacity of mitochondria, causing an accumulation of pyruvate, and thereby, an increase in lactate formation [46]. This is further potentiated by a decrease in lactate utilization [47]. Besides an alteration in the glycolytic pathway, tissue perfusion heterogeneity is being put forward as a possible reason for hyperlactatemia accompanied by physiological systemic $p \mathrm{O}_{2}$ during sepsis by Gutierrez and colleagues [18]. Under shock conditions, the blood circulation is centralized to vital organs, and non-vital tissues are characterized by a compromised peripheral vascular perfusion [48]. In our study, we clinically observed cyanosis of the extremities and dermographism in five out of 28 LPS-treated pigs (Tesch et al., 2015, submitted, [31]), as well as liver hemorrhage in LPS-treated animals (Renner et al., 2015, [49]). In contrast to our observations, other studies with a similar setup reported dermographism in most of the LPS-treated animals, as well as macroscopic and microscopic intrahepatic hyperperfusion [8]. These symptoms can be attributed, amongst others, to tissue perfusion heterogeneity and, thus, would fit the hypothesis voiced earlier [18]. Further, an increased lactic acid output of leukocytes due to an increased glycolysis has been suggested as a contributing factor to the lactic acidosis observed in septic animals [50]. In our study, leukocytes in particular were severely affected by LPS and an enhanced glycolysis, and thus, the output 
of lactic acid into the blood might have contributed to the present lactic acidosis (Tesch et al., 2015, submitted, [31]).

There was no difference between pre- or post-hepatic LPS infusion in CON-fed animals concerning the acid-base balance. An influence of DON was only observed in post-hepatic LPS-infused animals, whereas no dietary impact in pre-hepatic LPS-infused animals was observed regarding different acid-base balance variables in our trial. These results suggest a DON-related priming of post-hepatic cells involved in the exacerbation of metabolic disorders caused by LPS stimulation. Moreover, the lack of an assumed partial hepatic LPS clearance in post-hepatic LPS-infused DON-fed pigs compared to their pre-hepatic LPS-infused DON-fed counterparts might have triggered latent interactions between LPS and DON. Previously, it has been shown that an exposure to DON causes an altered immune response due to an upregulation of the transcriptional and post-transcriptional expression of cytokines, chemokines and inflammatory genes in porcine in vitro and in vivo studies [6-8]. In rodent studies, LPS priming of animals prior to DON exposure resulted in a stronger cytokine response compared to vehicle-treated animals [51], as well as simultaneous LPS and DON treatment of RAW264.7 macrophages [52]. However, we observed a uniform increase of TNF- $\alpha$ after 30 min with peak values at $60 \mathrm{~min}$ in all LPS-infused pigs, irrespective of the site of infusion or dietary treatment. Therefore, in our study, we could not confirm a superinduction of TNF- $\alpha$ in LPS-treated animals fed with a DON-contaminated diet (Tesch et al., 2015, submitted, [31]).

Similar to other studies that have investigated the influence of a systemic inflammatory response on blood glucose levels, we observed an initial hyperglycemia followed by eu- and hypoglycemia [21,53]. However, this kinetic was not significantly distinguishable from the post-prandial increase in blood glucose, which was observed in all groups as LPS infusion superimposed with the post-prandial effects.

The DON fed animals exhibited a markedly lower portal glucose level, which can most likely be attributed to the negative effects of DON on glucose transport across the intestinal barrier, as described previously by Halawa et al. [54]. This effect was also observed in several other studies using different animal and in vitro models. In chickens, DON inhibited the jejunal SGLT-1 activity (sodium-linked glucose transporter 1), responsible for active glucose uptake into enterocytes from lumen [55]. Furthermore, in human cell line HT-29, SGLT-1, GLUT-5 (glucose transporter 5, D-fructose associated) and GLUT-1 (passive D-glucose transporter) were inhibited by DON in a dose-dependent manner [56]. However, other studies in swine did not confirm this effect on SGLT-1 activity in brush border vesicles derived from the jejunum [57]. In addition to this DON effect, an additive effect of LPS infusion was detected. All LPS-infused groups had lower portal glucose concentrations 45 min after infusion start compared to the total control group. Generally under shock conditions, hypoperfusion of the intestinal tract can be observed [58,59], and therefore, it can be hypothesized that the transport capacity of the intestinal mucosa is impaired. Furthermore, in several LPS studies, an inhibitory effect on intestinal glucose transport was observed, for instance decreased GLUT-5 [60] and SGLT-1 [61] levels in LPS-treated rabbits. Furthermore, Amador and co-authors [62] observed an inhibitory effect of TNF- $\alpha$ on SGLT-1 ex vivo in rabbit's intestine. We could thus hypothesize that in our study, the impaired portal glucose uptake in LPS-treated animals (45 min-180 min) might be, at least partially, attributed to an impairment in SGLT-1 transport capacity due to the increase in TNF- $\alpha$ (Tesch et al., 2015, submitted, [31]).

Our data suggest that chronic oral exposure to DON exacerbates lactic acidosis by a post-hepatic LPS-induced systemic inflammation, while a pre-hepatic LPS stimulation did not result in 
such amplification. This different responsiveness between pre- and post-hepatic-infused animals was not observed within the control-fed groups.

\section{Experimental Section}

Animal experiments were conducted according to the EC regulations concerning the protection of experimental animals and the guidelines of the German Animal Welfare Act approved by the Lower Saxony State Office for Consumer Protection and Food Safety (Lower Saxony State Office for Consumer Protection and Food Safety; File Number 33.4-42502-04-13/1274).

\subsection{Experimental Design and Procedures}

A total of 41 barrows (German landrace, Mariensee, Germany) were randomly assigned to either a group receiving natural DON-contaminated feed (DON; $4.59 \mathrm{mg} \mathrm{DON} / \mathrm{kg}$ feed; $n=19$ ) or a control group (CON; $n=22$ ) control diet (Table 3$)$. Experimental groups, their treatment and the number of animals are illustrated in Figure 9. The pigs had an average initial weight of $25.8 \pm 3.7 \mathrm{~kg}$ (means \pm SD) and were fed restrictively with 2 single portions of $700 \mathrm{~g}$ per day, mixed with water and provided as mash. All barrows were housed in individual floor pens during the first 21 days of the trial and subsequently transferred into individual metabolism crates (described in [63]) until Day 29.

Table 3. Diet composition, based on air dry matter $(\mathrm{ADM})=88.37 \%$.

\begin{tabular}{ccc}
\hline Ingredients & CON \% & DON \% \\
\hline barley & 53.30 & 53.30 \\
maize (non contaminated) & 15.00 & 7.50 \\
maize (contaminated) & - & 7.50 \\
soybean meal & 20.00 & 20.00 \\
rapeseed & 5.00 & 5.00 \\
soybean oil & 2.00 & 2.00 \\
Premix ${ }^{1}$ & 3.00 & 3.00 \\
Lysine-HCl & 0.40 & 0.40 \\
L-Threonine & 0.12 & 0.12 \\
DL-Methionine & 0.15 & 0.15 \\
HCl-insoluble ash ${ }^{2}$ & 1.00 & 1.00 \\
analysed composition & $\mathrm{g} / \mathrm{kg} \mathrm{ADM}$ & $\mathrm{g} / \mathrm{kg} \mathrm{ADM}$ \\
crude protein & 196.85 & 194.83 \\
crude fat & 47.48 & 46.51 \\
crude ash & 69.70 & 69.51 \\
crude fiber & 51.26 & 49.32 \\
deoxynivalenol mg/kg & 0.12 & 4.59 \\
\hline
\end{tabular}

${ }^{1}$ Provided per kilogram of premix: Ca 25 g, P 6 g, Na 5.5 g, Mg 1 g, Fe 4000 mg, Cu 500 mg, Mn 2670 mg, Zn 3340 mg, I 67 mg, Se 13.5 mg, Co 8.3 mg, bas. Co-II-carb-monohydrat $8.3 \mathrm{mg}$, vitamin A 400000 I.U., vitamin D3 40000 I.U., vitamin E $1200 \mathrm{mg}$, vitamin $B_{1} 37.5 \mathrm{mg}$, vitamin $B_{2} 100 \mathrm{mg}$, vitamin $\mathrm{B}_{6} 100 \mathrm{mg}$, vitamin $\mathrm{B}_{12} 750 \mu \mathrm{g}$, vitamin K3 $52.5 \mathrm{mg}$, nicotinic acid $500 \mathrm{mg}$, pantothenic acid $337.5 \mathrm{mg}$, choline chloride $5,000 \mathrm{mg} ;{ }^{2}>97 \% \mathrm{SiO}_{2}$ (Sipernat ${ }^{\mathbb{B}} 22 \mathrm{~S}$, Evonic Industries, Hanau-Wolfgang, Germany). 
In order to facilitate pre- or post-hepatic blood-sampling and infusion, pigs were surgically fitted with 5 differently-located, permanent indwelling catheters under general inhalation anesthesia (Isoflurane ${ }^{\circledR}$, CP-Pharma, Burgdorf, Germany) at Day 27 of the trial when the animals had an average body weight of $40.5 \pm 3.0 \mathrm{~kg}$. Permanent Silastic ${ }^{\circledR}$ catheters were manufactured from Dow Corning (Midland, TX, USA) medical-grade tubing material (1.57 $\mathrm{mm}$ ID and $3.18 \mathrm{~mm}$ OD), autoclaved and placed in the Vena jugularis interna, Vena jugularis externa, Vena lienalis, Vena portae hepatis and Arteria carotis communis. Catheters were tunneled to the neck and left flank, respectively, and fixed with catheter mounts/clamps (Arrow, Teleflex Medical GmbH, Kernen, Germany) on the skin. Catheters were fitted with three-way valves (Walther-CMP, Kiel, Germany) for sampling and flushed with heparinized physiological saline (1 mL sodium heparin (25.000 IE/5 mL) (Ratiopharm, Ulm, Germany); dissolved in $500 \mathrm{~mL}$ sterile $0.9 \% \mathrm{NaCl}$ (B. Braun Melsungen AG, Melsungen, Germany) every $4 \mathrm{~h}$ and after each sampling to prevent blood coagulation. Two days between surgery and sampling day were allowed for recovery. Throughout this recovery period, half of the daily ration was fed ( 2 times $350 \mathrm{~g} /$ day).

At Day 29 of the trial, animals were further divided into 3 infusion groups: LPS pre-hepatic (LPS portal- $\left.\mathrm{CON}_{\text {jugular }}\right)$, LPS post-hepatic $\left(\mathrm{CON}_{\text {portal }}-\mathrm{LPS}_{\text {jugular }}\right)$ or control $\left(\mathrm{CON}_{\text {portal- }} \mathrm{CON}_{\text {jugular }}\right)$, illustrated in Figure 9.

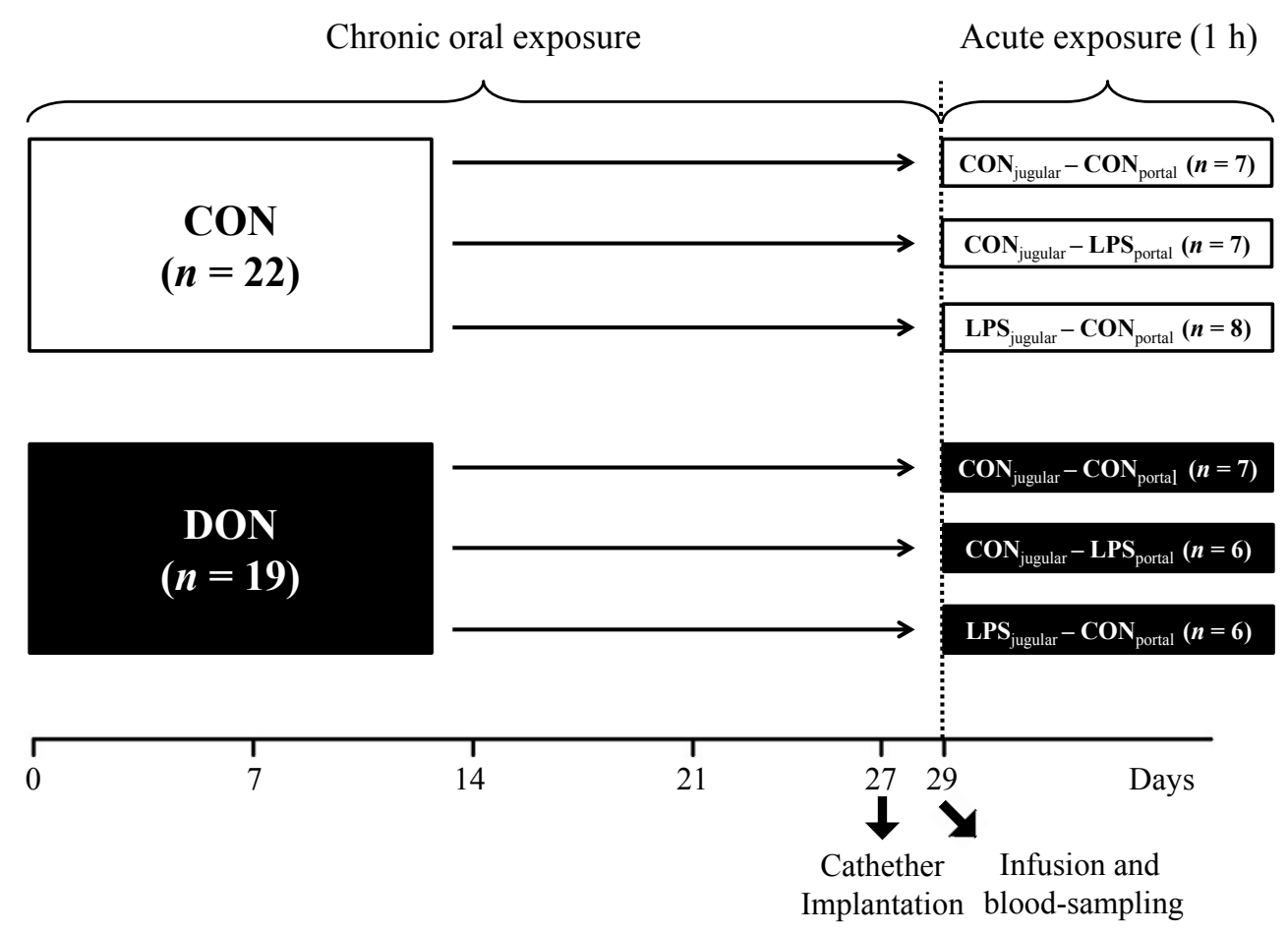

Figure 9. Experimental design.

On the sampling day, the time of infusion start was set as zero, and blood samples were taken at -30 , 15, 30, 45, 60, 75, 90, 120, 150 and 180 min from Vena jugularis interna, Vena portae hepatis and Arteria carotis communis. Fifteen minutes prior to infusion, pigs received $700 \mathrm{~g}$ of feed each. LPS was infused at $7.5 \mu \mathrm{g} \mathrm{LPS} / \mathrm{kg}$ BW for $1 \mathrm{~h}$ (Escherichia coli-LPS, O111:B4, Sigma-Aldrich, Taufkirchen, Germany), and $0.9 \% \mathrm{NaCl}$ was used as the control substance. Infusion was implemented using an infusion pump (IPC-N-4, ISMATEC Laboratoriumstechnik GmbH, Wertheim, Germany) and infusion tubes with a $2.06 \mathrm{~mm}$ inner diameter (PharMed ${ }^{\circledR}$ Ismaprene, Wertheim, Germany, ISMATEC), 
administered into either Vena jugularis externa or Vena lienalis. The pigs were slaughtered $195 \mathrm{~min}$ after infusion start.

\subsection{Sample Analysis}

For a red hemogram assessment, 1-mL blood samples were collected in EDTA tubes and analyzed immediately with an automated hematology analyzer (Celltac alpha MEK-6450, Nihon Kohden Corporation, Tokyo, Japan). Furthermore, blood samples $(0.5 \mathrm{~mL})$ for blood gases, electrolytes, $\mathrm{pH}$, glucose and lactate were collected into blood sample syringes (SC-Sanguis Counting GmbH, Nümbrecht, Germany), and variables were assessed immediately using an automated blood gas and electrolyte analyzer (GEM Premier 4000, Werfen, Kirchheim, Germany). Anion gap and base excess were calculated using the equations detailed below.

\subsection{Calculations}

Equation used to calculate anion gap (AG) [64]:

$$
A G=\left(\mathrm{Na}^{+}+\mathrm{K}^{+}\right)-\left(\mathrm{Cl}^{-}+\mathrm{HCO}_{3}^{-}\right)
$$

$\mathrm{Na}^{+}=$sodium; $\mathrm{K}^{+}=$potassium; $\mathrm{Cl}^{-}=$chloride $; \mathrm{HCO}_{3}{ }^{-}=$bicarbonate .

Equation used to calculate base excess (BE) [65]:

$$
\mathrm{BE}=(1-0.0143 \times \mathrm{cHb})
$$

$$
\begin{gathered}
\times\left[\left(0.0304 \times p \mathrm{CO}_{2} \times 10^{\mathrm{pH}-6.1}-24.26\right)+(9.5+1.63 \times c H b) \times(\mathrm{pH}-7.4)\right] \\
-0.2 \times \mathrm{cHb} \times\left(1-\mathrm{sO}_{2}\right)
\end{gathered}
$$

$\mathrm{cHb}=$ total hemoglobin concentration; $p \mathrm{CO}_{2}=$ carbon dioxide partial pressure; $s \mathrm{O}_{2}=$ oxygen saturation .

\subsection{Statistical Analysis}

Data were evaluated by using PROC MIXED in SAS Enterprise Guide 6.1 (SAS Institute 2013, Cary, NC, USA) using a restricted maximum likelihood model (REML). Group, catheter, time and their interaction were defined as fixed factors. A "REPEATED" statement was included to account for the individual similarity at repeated measurements. The "compound symmetry" was found to be the most appropriate co-variance structure according to the corrected Akaike's information criterion (AICC), and significant effects at different time points were further evaluated by multiple $t$-tests ("pairwise differences" (PDIFF)). Results are presented as least square means (LSMeans) and pooled standard error of means (PSEM).

\section{Conclusions}

Concluding, we could demonstrate chronic enteral DON-exposure had a definite priming effect on the pig's organism, specifically the acid-base balance, thus aggravating the sole impact of LPS depending on the site of its entry. The involvement of the liver in this scenario was apparent as post-hepatic LPS exposure elicited a much stronger impact compared to pre-hepatic exposure in combination with mycotoxin feeding. Portal glucose uptake was significantly diminished in DON-fed 
animals compared to control, thus giving in vivo evidence to the previously-reported impairment of glucose transporter activity in ex vivo studies.

\section{Acknowledgments}

This study was financially supported by Deutsche Forschungsgemeinschaft (DFG DA 558/1-4 and RO 743/3-3). The authors would like to thank Nicola Mickenautsch, Lara Lindner and Elenia Scholz for their excellent technical support in sample analysis. Furthermore, the authors gratefully acknowledge Melanie Schären for critical language editing of the manuscript.

\section{Author Contributions}

Head of organization and execution: Erik Bannert, Tanja Tesch, Jeannette Kluess, Sven Dänicke, Jana Frahm; Trial and project design: Sven Dänicke, Jeannette Kluess, Susanne Kersten, Jana Frahm, Hermann-Josef Rothkötter; Trial implementation and sample collection: Erik Bannert, Tanja Tesch, Jeannette Kluess, Sven Dänicke, Stefan Kahlert, Lydia Renner, Jana Frahm; Data analysis and interpretation: Erik Bannert, Jeannette Kluess, Sven Dänicke, Jana Frahm; Writing of manuscript: Erik Bannert; Revision of manuscript: Sven Dänicke, Jeannette Kluess, Hermann-Josef Rothkötter, Stefan Kahlert, Lydia Renner, Jana Frahm, Tanja Tesch.

\section{Conflicts of Interest}

The authors declare no conflict of interest.

\section{References}

1. European Food Safety Authority. Deoxynivalenol in food and feed: Occurrence and exposure. EFSA J. 2013, 10, 56, doi:10.2903/j.efsa.2013.3379.

2. Bondy, G.S.; Pestka, J.J. Immunomodulation by fungal toxins. J. Toxicol. Environ. Health $B$ Crit. Rev. 2000, 3, 109-143.

3. Rotter, B.A.; Prelusky, D.B.; Pestka, J.J. Toxicology of deoxynivalenol (vomitoxin). J. Toxicol. Environ. Health B Crit. Rev. 1996, 48, 1-34.

4. Hannon, J.P. Blood acid-base curve nomogram for immature domestic pigs. Am. J. Vet. Res. 1983, 44, 2385-2390.

5. Pestka, J.J.; Zhou, H.R.; Moon, Y.; Chung, Y.J. Cellular and molecular mechanisms for immune modulation by deoxynivalenol and other trichothecenes: Unraveling a paradox. Toxicol. Lett. 2004, 153, 61-73.

6. Döll, S.; Schrickx, J.A.; Dänicke, S.; Fink-Gremmels, J. Deoxynivalenol-induced cytotoxicity, cytokines and related genes in unstimulated or lipopolysaccharide stimulated primary porcine macrophages. Toxicol. Lett. 2009, 184, 97-106.

7. Dänicke, S.; Brosig, B.; Kersten, S.; Kluess, J.; Kahlert, S.; Panther, P.; Diesing, A.K.; Rothkötter, H.J. The Fusarium toxin deoxynivalenol (DON) modulates the LPS induced acute phase reaction in pigs. Toxicol. Lett. 2013, 220, 172-180. 
8. Stanek, C.; Reinhardt, N.; Diesing, A.K.; Nossol, C.; Kahlert, S.; Panther, P.; Kluess, J.; Rothkötter, H.J.; Kuester, D.; Brosig, B.; et al. A chronic oral exposure of pigs with deoxynivalenol partially prevents the acute effects of lipopolysaccharides on hepatic histopathology and blood clinical chemistry. Toxicol. Lett. 2012, 215, 193-200.

9. Döll, S.; Schrickx, J.A.; Dänicke, S.; Fink-Gremmels, J. Interactions of deoxynivalenol and lipopolysaccharides on cytokine excretion and mRNA expression in porcine hepatocytes and Kupffer cell enriched hepatocyte cultures. Toxicol. Lett. 2009, 190, 96-105.

10. Döll, S.; Schrickx, J.A.; Valenta, H.; Dänicke, S.; Fink-Gremmels, J. Interactions of deoxynivalenol and lipopolysaccharides on cytotoxicity protein synthesis and metabolism of DON in porcine hepatocytes and Kupffer cell enriched hepatocyte cultures. Toxicol. Lett. 2009, 189, 121-129.

11. Salomao, R.; Brunialti, M.K.; Rapozo, M.M.; Baggio-Zappia, G.L.; Galanos, C.; Freudenberg, M. Bacterial sensing, cell signaling, and modulation of the immune response during sepsis. Shock 2012, 38, 227-242.

12. Cinel, I.; Dellinger, R.P. Advances in pathogenesis and management of sepsis. Curr. Opin. Infect. Dis. 2007, 20, 345-352.

13. Marshall, J.C. Endotoxin in the pathogenesis of sepsis. Contrib. Nephrol. 2010, 167, 1-13.

14. Vincent, J.L.; Rello, J.; Marshall, J.; Silva, E.; Anzueto, A.; Martin, C.D.; Moreno, R.; Lipman, J.; Gomersall, C.; Sakr, Y.; et al. International study of the prevalence and outcomes of infection in intensive care units. JAMA 2009, 302, 2323-2329.

15. Martin, G.S.; Mannino, D.M.; Eaton, S.; Moss, M. The epidemiology of sepsis in the United States from 1979 through 2000. N. Engl. J. Med. 2003, 348, 1546-1554.

16. Wyns, H.; Plessers, E.; de Backer, P.; Meyer, E.; Croubels, S. In vivo porcine lipopolysaccharide inflammation models to study immunomodulation of drugs. Vet. Immunol. Immunopathol. 2015, 10.1016/j.vetimm.2015.06.001.

17. Angus, D.C.; van der Poll, T. Severe sepsis and septic shock. N. Engl. J. Med. 2013, 369, 840-895.

18. Gutierrez, G.; Wulf, M.E. Lactic acidosis in sepsis: A commentary. Intensive Care Med. 1996, 22, 6-16.

19. Gibot, S. On the origins of lactate during sepsis. Crit. Care Med. 2012, 16, 151, doi:10.1186/cc11472.

20. Bakker, J.; Coffernils, M.; Leon, M.; Gris, P.; Vincent, J.L. Blood lactate levels are superior to oxygen-derived variables in predicting outcome in human septic shock. Chest 1991, 99, 956-962.

21. Maitra, S.R.; Wojnar, M.M.; Lang, C.H. Alterations in tissue glucose uptake during the hyperglycemic and hypoglycemic phases of sepsis. Shock 2000, 13, 379-385.

22. Gibaldi, M.; Boyes, R.N.; Feldman, S. Influence of first-pass effect on availability of drugs on oral administration. J. Pharm. Sci. 1971, 60, 1338-1340.

23. Jirillo, E.; Caccavo, D.; Magrone, T.; Piccigallo, E.; Amati, L.; Lembo, A.; Kalis, C.; Gumenscheimer, M. The role of the liver in the response to LPS: Experimental and clinical findings. J. Endotoxin Res. 2002, 8, 319-327.

24. Yan, J.; Li, S.; Li, S. The role of the liver in sepsis. Int. Rev. Immunol. 2014, 33, 498-510.

25. Tomlinson, J.E.; Blikslager, A.T. Interactions between lipopolysaccharide and the intestinal epithelium. J. Am. Vet. Med. Assoc. 2004, 224, 1446-1452. 
26. Kraft, W.; Dürr, U.M.; Moritz, A. Klinische Labordiagnostik in der Tiermedizin; Schattauer Verlag: Germany, 2014; pp. 507-516.

27. Jackson, P.; Cockcroft, P. Clinical Examination of Farm Animals; Wiley-Blackwell: Malden, MA, USA, 2002; pp. 301-305.

28. Bickhardt, K.; Wirtz, A. The influence of stress by restraint and of feeding on blood values of pigs. AGRIS 1978, 12, 457-462.

29. Bone, R.C.; Balk, R.A.; Cerra, F.B.; Dellinger, R.P.; Fein, A.M.; Knaus, W.A.; Schein, R.M.; Sibbald, W.J. Definitions for sepsis and organ failure and guidelines for the use of innovative therapies in sepsis. The ACCP/SCCM Consensus Conference Committee. American College of Chest Physicians/Society of Critical Care Medicine. Chest 1992, 101, 1644-1655.

30. Jones, G.R.; Lowes, J.A. The systemic inflammatory response syndrome as a predictor of bacteraemia and outcome from sepsis. QJM 1996, 89, 515-522.

31. Tesch, T.; Bannert, E.; Kluess, J.; Frahm, J.; Kersten, S.; Breves, G.; Renner, L.; Kahlert, S.; Rothkötter, H.J.; Dänicke, S. Does dietary deoxynivalenol modulates the acute phase reaction in endotoxaemic pigs?-Lessons from clinical signs, white blood cell counts and TNF-alpha. Toxins 2015, in press.

32. McLellan, T.M. The influence of a respiratory acidosis on the exercise blood lactate response. Eur. J. Appl. Physiol. Occup. Physiol. 1991, 63, 6-11.

33. Irizarry, R.; Reiss, A. Arterial and venous blood gases: Indications, interpretations, and clinical applications. Compend. Contin. Educ. Pract. Vet. 2009, 31, E1-E7.

34. Grenier, B.; Loureiro-Bracarense, A.P.; Lucioli, J.; Pacheco, G.D.; Cossalter, A.M.; Moll, W.D.; Schatzmayr, G.; Oswald, I.P. Individual and combined effects of subclinical doses of deoxynivalenol and fumonisins in piglets. Mol. Nutr. Food Res. 2011, 55, 761-771.

35. Modra, H.; Blahova, J.; Marsalek, P.; Banoch, T.; Fictum, P.; Svoboda, M. The effects of mycotoxin deoxynivalenol (DON) on haematological and biochemical parameters and selected parameters of oxidative stress in piglets. Neuroendocrinol. Lett. 2013, 34, 84-89.

36. Zielonka, L.; Gajecka, M.; Tarasiuk, M.; Gajecki, M. The effects of dietary deoxynivalenol (DON) on selected blood biochemical and hematological parameters in pre-pubertal gilts. Pol. J. Vet. Sci. 2015, 18, 223-231.

37. Suri, M.; Kumar, L.; Kaur, G.; Singhi, S.; Prasad, R. Electrolyte disturbances due to ouabain sensitive sodium potassium pump in erythrocytes of children with sepsis. Indian J. Med. Res. 1997, $105,67-71$.

38. Illner, H.; Shires, G.T. Changes in sodium, potassium, and adenosine-triphosphate contents of red-blood-cells in sepsis and septic shock. Circ. Shock 1982, 9, 259-267.

39. Shires, G.T., 3rd; Peitzman, A.B.; Illner, H.; Shires, G.T. Changes in red blood cell transmembrane potential, electrolytes, and energy content in septic shock. J. Trauma 1983, 23, 769-774.

40. Sayeed, M.M. Ion transport in circulatory and/or septic shock. Am. J. Physiol. 1987, 252, R809-R821.

41. Hotchkiss, R.S.; Song, S.K.; Ling, C.S.; Ackerman, J.J.; Karl, I.E. Sepsis does not alter red blood cell glucose metabolism or $\mathrm{Na}+$ concentration: A 2H-, 23Na-NMR study. Am. J. Physiol. 1990, 258, R21-R31. 
42. James, J.H.; Fang, C.H.; Schrantz, S.J.; Hasselgren, P.O.; Paul, R.J.; Fischer, J.E. Linkage of aerobic glycolysis to sodium-potassium transport in rat skeletal muscle-Implications for increased muscle lactate production in sepsis. J. Clin. Investig. 1996, 98, 2388-2397.

43. Hurtado, F.J.; Gutierrez, A.M.; Silva, N.; Fernandez, E.; Khan, A.E.; Gutierrez, G. Role of tissue hypoxia as the mechanism of lactic-acidosis during Escherichia coli endotoxemia. J. Appl. Physiol. 1992, 72, 1895-1901.

44. Boekstegers, P.; Weidenhofer, S.; Kapsner, T.; Werdan, K. Skeletal muscle partial pressure of oxygen in patients with sepsis. Crit. Care Med. 1994, 22, 640-650.

45. Revelly, J.P.; Tappy, L.; Martinez, A.; Bollmann, M.; Cayeux, M.C.; Berger, M.M.; Chiolero, R.L. Lactate and glucose metabolism in severe sepsis and cardiogenic shock. Crit. Care Med. 2005, 33, 2235-2240.

46. Robinson, B.H. Lacticacidemia. Biochim. Biophys. Acta 1993, 1182, 231-244.

47. Levraut, J.; Ciebiera, J.P.; Chave, S.; Rabary, O.; Jambou, P.; Carles, M.; Grimaud, D. Mild hyperlactatemia in stable septic patients is due to impaired lactate clearance rather than overproduction. Am. J. Respir. Crit. Care Med. 1998, 157, 1021-1026.

48. Schumer, W. Pathophysiology and treatment of septic shock. Am. J. Emerg. Med. 1984, 2, 74-77.

49. Renner, L.; Kahlert, S.; Bannert, E.; Tesch, T.; Kluess, J.; Kersten, S.; Dänicke, S.; Rothkötter, H.J. Effects of chronic DON exposure and systemic LPS administration on liver histopathology in pigs. In Proceedings of the 37th Mycotoxin Workshop, Bratislava, Slovakia, 1-3 June 2015.

50. Haji-Michael, P.G.; Ladriere, L.; Sener, A.; Vincent, J.L.; Malaisse, W.J. Leukocyte glycolysis and lactate output in animal sepsis and ex vivo human blood. Metabolism 1999, 48, 779-785.

51. Islam, Z.; Pestka, J.J. LPS priming potentiates and prolongs proinflammatory cytokine response to the trichothecene deoxynivalenol in the mouse. Toxicol. Appl. Pharmacol. 2006, 211, 53-63.

52. Wong, S.S.; Zhou, H.R.; Marin-Martinez, M.L.; Brooks, K.; Pestka, J.J. Modulation of IL-1ß, IL-6 and TNF- $\alpha$ secretion and mRNA expression by the trichothecene vomitoxin in the RAW 264.7 murine macrophage cell line. Food Chem. Toxicol. 1998, 36, 409-419.

53. Lang, C.H.; Spolarics, Z.; Ottlakan, A.; Spitzer, J.J. Effect of high-dose endotoxin on glucose-production and utilization. Metabolism 1993, 42, 1351-1358.

54. Halawa, A.; Dänicke, S.; Kersten, S.; Breves, G. Effects of deoxynivalenol and lipopolysaccharide on electrophysiological parameters in growing pigs. Mycotoxin Res. 2012, 28, 243-252.

55. Awad, W.A.; Aschenbach, J.R.; Setyabudi, F.M.; Razzazi-Fazeli, E.; Bohm, J.; Zentek, J. In vitro effects of deoxynivalenol on small intestinal D-glucose uptake and absorption of deoxynivalenol across the isolated jejunal epithelium of laying hens. Poult. Sci. 2007, 86, 15-20.

56. Maresca, M.; Mahfoud, R.; Garmy, N.; Fantini, J. The mycotoxin deoxynivalenol affects nutrient absorption in human intestinal epithelial cells. J. Nutr. 2002, 132, 2723-2731.

57. Zerull, K.; Breves, G.; Schroder, B.; Goyarts, B.; Dänicke, S. The influence of the mycotoxin deoxynivalenol on jejunal glucose transport in pigs. Mycotoxin Res. 2005, 21, 251-257.

58. Vallet, B.; Lund, N.; Curtis, S.E.; Kelly, D.; Cain, S.M. Gut and muscle tissue $p \mathrm{O}_{2}$ in endotoxemic dogs during shock and resuscitation. J. Appl. Physiol. 1994, 76, 793-800.

59. Whitworth, P.W.; Cryer, H.M.; Garrison, R.N.; Baumgarten, T.E.; Harris, P.D. Hypoperfusion of the intestinal microcirculation without decreased cardiac output during live Escherichia coli sepsis in rats. Circ. Shock 1989, 27, 111-122. 
60. Garcia-Herrera, J.; Marca, M.C.; Brot-Laroche, E.; Guillen, N.; Acin, S.; Navarro, M.A.; Osada, J.; Rodriguez-Yoldi, M.J. Protein kinases, TNF-alpha, and proteasome contribute in the inhibition of fructose intestinal transport by sepsis in vivo. Am. J. Physiol. Gastrointest. Liver Physiol. 2008, 294, G155-G164.

61. Amador, P.; Garcia-Herrera, J.; Marca, M.C.; de la Osada, J.; Acin, S.; Navarro, M.A.; Salvador, M.T.; Lostao, M.P.; Rodriguez-Yoldi, M.J. Intestinal D-galactose transport in an endotoxemia model in the rabbit. J. Membr. Biol. 2007, 215, 125-133.

62. Amador, P.; Garcia-Herrera, J.; Marca, M.C.; de la Osada, J.; Acin, S.; Navarro, M.A.; Salvador, M.T.; Lostao, M.P.; Rodriguez-Yoldi, M.J. Inhibitory effect of TNF- $\alpha$ on the intestinal absorption of galactose. J. Cell. Biochem. 2007, 101, 99-111.

63. Farries, F.E.; Oslage, H.J. Zur Technik langfristiger Stoffwechselversuche an wachsenden Schweinen. Z. Tierphysiol. Tierernähr. Futtermittelkd. 1961, 16, 11-19.

64. Emmett, M.; Narins, R.G. Clinical Use of Anion Gap. Medicine 1977, 56, 38-54.

65. Lang, W.; Zander, R. The accuracy of calculated base excess in blood. Clin. Chem. Lab. Med. 2002, 40, 404-410.

(C) 2015 by the authors; licensee MDPI, Basel, Switzerland. This article is an open access article distributed under the terms and conditions of the Creative Commons Attribution license (http://creativecommons.org/licenses/by/4.0/). 